TRANSACTIONS OF THE

AMERICAN MATHEMATICAL SOCIETY

Volume 348, Number 5, May 1996

\title{
THE BERGMAN KERNEL FUNCTION OF SOME REINHARDT DOMAINS
}

\author{
SHENG GONG AND XUEAN ZHENG
}

Abstract. The boundary behavior of the Bergman Kernel function of some Reinhardt domains is studied. Upper and lower bounds for the Bergman kernel function are found at the diagonal points $(z, \bar{z})$. Let $D$ be the Reinhardt domain

$$
D=\left\{\left.z \in \mathbf{C}^{n}\left|\|z\|_{\alpha}=\sum_{j=1}^{n}\right| z_{j}\right|^{2 / \alpha_{j}}<1\right\}
$$

where $\alpha_{j}>0, j=1,2, \ldots, n$; and let $K(z, \bar{w})$ be the Bergman kernel function of $D$. Then there exist two positive constants $m$ and $M$ and a function $F$ such that

$$
m F(z, \bar{z}) \leq K(z, \bar{z}) \leq M F(z, \bar{z})
$$

holds for every $z \in D$. Here

$$
F(z, \bar{z})=(-r(z))^{-n-1} \prod_{j=1}^{n}\left(-r(z)+\left|z_{j}\right|^{2 / \alpha_{j}}\right)^{1-\alpha_{j}}
$$

and $r(z)=\|z\|_{\alpha}-1$ is the defining function for $D$. The constants $m$ and $M$ depend only on $\alpha=\left(\alpha_{1}, \ldots, \alpha_{n}\right)$ and $n$, not on $z$.

\section{INTRODUCTION}

The Bergman kernel function $K(z, \bar{w}), z, w \in \Omega$ for a domain $\Omega \subset \mathbf{C}^{n}$ is the kernel of the Bergman projection operator, the operator projecting $L^{2}(\Omega)$ onto its holomorphic subspace. In this paper, we consider the Reinhardt domain

$$
D=\left\{\left.z \in \mathbf{C}^{n}\left|\|z\|_{\alpha}=\sum_{j=1}^{n}\right| z_{j}\right|^{2 / \alpha_{j}}<1\right\}
$$

where $\alpha_{j}>0, j=1,2, \ldots, n$. The purpose of this paper is to give an estimate of the kernel function $K(z, \bar{z})$ of $D$ in a "small constant-large constant" sense. Precisely, we prove the following

Theorem 1. There exist two positive constants $m$ and $M$, which only depend on $n$ and $\alpha_{j}, j=1,2, \ldots, n$, such that

$$
m F(z, \bar{z}) \leq K(z, \bar{z}) \leq M F(z, \bar{z})
$$

Received by the editors October 13, 1994.

1991 Mathematics Subject Classification. Primary 32H10.

(C)1996 American Mathematical Society 
holds for every $z \in D$, where

$$
F(z, \bar{z})=(-r(z))^{-n-1} \prod_{j=1}^{n}\left(-r(z)+\left|z_{j}\right|^{2 / \alpha_{j}}\right)^{1-\alpha_{j}}
$$

and $r(z)=\|z\|_{\alpha}-1$ is the defining function for $D$.

The study of the boundary behavior of $K_{\Omega}(z, \bar{w})$ for a domain $\Omega \subset \mathbf{C}^{n}$ is quite old, going back to the original inquires of Bergman [1]. Both Fefferman [6] and later, Boutet de Monvel and Sjöstrand [2] obtained the asymptotic expansion of $K_{\Omega}(z, \bar{w})$, when $\Omega \Subset \mathbf{C}^{n}$ is strongly pseudo-convex. For domains in $\mathbf{C}^{2}$, Catlin [3] has given a precise description of the behavior of $K_{\Omega}(z, \bar{z})$ near finite-type points in $\partial \Omega$. Sharp estimates for $K_{\Omega}(z, \bar{w})$ for this class of domains were obtained by McNeal [7] and Nagel et al. [9]. For a "decoupled" class of $\Omega \Subset \mathbf{C}^{n}$, McNeal [8] described the exact estimates on $K_{\Omega}(z, \bar{w})$ for $z$ near a point of finite type in $\partial \Omega$. For the Reinhardt domain (1), D'Angelo [4] gives the series form of the Bergman kernel function $K(z, \bar{w})$ as

$$
\begin{aligned}
& K(z, \bar{w})=\left(\alpha_{1} \alpha_{2} \cdots \alpha_{n} \pi^{n}\right)^{-1} \\
& \quad \times \sum_{m_{1}, m_{2}, \ldots, m_{n} \geq 0} \frac{\Gamma\left(\sum_{j=1}^{n} \alpha_{j}\left(m_{j}+1\right)+1\right)}{\Gamma\left(\alpha_{1} m_{1}+\alpha_{1}\right) \cdots \Gamma\left(\alpha_{n} m_{n}+\alpha_{n}\right)}\left(z_{1} \bar{w}_{1}\right)^{m_{1}} \cdots\left(z_{n} \bar{w}_{n}\right)^{m_{n}} .
\end{aligned}
$$

We will use equation (4) to obtain the estimate of $K(z, \bar{z})$ given in inequalities (2).

It should be noted that D'Angelo $[4,5]$ has studied $K(z, \bar{z})$ for certain domains. He showed

$$
K(z, \bar{z})=\sum_{k=0}^{l+1} c_{k}\left(1-\sum_{j=1}^{l}\left|z_{j}\right|^{2}\right)^{-l-1+k p}\left(\left(1-\sum_{J=1}^{l}\left|z_{j}\right|^{2}\right)^{p}-\sum_{j=l+1}^{n}\left|z_{j}\right|^{2}\right)^{-n+l-k}
$$

when the domain is $\Omega=\left\{\left.z \in \mathbf{C}^{n}\left|\sum_{j=1}^{l}\right| z_{j}\right|^{2}+\left(\sum_{j=l+1}^{n}\left|z_{j}\right|^{2}\right)^{1 / p}<1\right\}$, where $1 \leq l<n, p$ is a positive real number, and the constants $c_{k}$ depend on $k, l, n$ and $p$ only.

The sketch of the proof is now indicated. We will informally use the word "comparable" to mean the two functions or sequences of coefficients are related by inequalities such as those between $K$ and $F$ given in inequalities (2). The proof can be outlined as follows: We start with the expansion of the Bergman kernel function in (4). Then we show that the coefficients are comparable to certain coefficients $A$ that are more tractable. The Main Lemma shows that these coefficients $A$ are comparable to certain coefficients $B$. It follows that the coefficients in equation (4) are comparable to the coefficients $B$. Multiplying by the powers of $z$ and summing, we find that the kernel function is comparable to a function $F$ which has an expansion with the coefficients $B$.

Most of the lemmas are combinatorial. The Main Lemma which relates the coefficients $A$ and $B$ is stated in section 3. Its proof is carried out in sections 4 and 5. The proof of the Theorem is given in sections 6 and 7 .

\section{Preliminary Lemmas}

We start with some combinatorial lemmas proved using generating function. The routine proofs are not included. 
Let $\mathbf{R}_{+}^{n}$ denote the set of vectors $x=\left(x_{1}, \ldots, x_{n}\right)$ in $\mathbf{R}^{n}$, such that all the components $x_{j}, j=1,2, \ldots, n$, are non-negative, and let $\sigma(x)=\sum_{i=1}^{n} x_{i}$. As usual we let $e_{j}$ denote the unit vector of the $j$-th coordinate in $\mathbf{R}^{n}$, and let $x(j)=$ $\left(x_{1}, \ldots, x_{j-1}, 0, x_{j+1}, \ldots, x_{n}\right), \sigma(x(j))=\sum_{i \neq j} x_{i}$ if $x=\left(x_{1}, \ldots, x_{n}\right)$.

Let $a, t$ be real numbers, $|t|<1$, and

$$
(1-t)^{-a}=\sum_{k=0}^{\infty} b(a, k) t^{k}
$$

\section{Lemma 1.}

$$
b(a, k)=\frac{\Gamma(k+a)}{\Gamma(a) \Gamma(k+1)}, \quad \text { when } a \neq 0, a>-1 ;
$$

and $b(0,0)=1, b(0, k)=0$ when $k \neq 0$.

Let $u=\left(u_{1}, \ldots, u_{n}\right) \in \mathbf{R}_{+}^{n}, \sigma(u)=\sum_{i=1}^{n} u_{i}<1$, and

$$
(1-\sigma(u))^{-a}=\sum_{m \in \mathbf{Z}_{+}^{n}} C(a, m) u^{m}
$$

where $m=\left(m_{1}, \ldots, m_{n}\right), u^{m}=u_{1}^{m_{1}} \cdots u_{n}^{m_{n}}$.

\section{Lemma 2.}

$$
C(a, m)=\frac{\Gamma(a+\sigma(m))}{\Gamma(a) \prod_{j=1}^{n} \Gamma\left(1+m_{j}\right)}, \quad \text { when } a \neq 0, a>-1
$$

and $C(0,0)=1, C(0, m)=0$ when $m \neq 0=(0, \ldots, 0)$.

Lemma 3. Let $a_{j}, j=1, \ldots, s$, be real numbers, $k \in \mathbf{Z}_{+}$, then

$$
b\left(a_{1}+\cdots+a_{s}, k\right)=\sum_{\sigma(l)=k} b\left(a_{1}, l_{1}\right) b\left(a_{2}, l_{2}\right) \cdots b\left(a_{s}, l_{s}\right)
$$

where $b(a, k)$ is defined by $(5), l=\left(l_{1}, \ldots, l_{s}\right) \in \mathbf{Z}_{+}^{s}$, and $\sigma(l)=\sum_{j=1}^{n} l_{j}$.

Lemma 4. Let $a_{j}, j=1, \ldots, s$, be real numbers, $l \in \mathbf{Z}_{+}^{n}$, then

$$
C\left(a_{1}+\cdots+a_{s}, l\right)=\sum_{m+p+\cdots+q=l} C\left(a_{1}, m\right) C\left(a_{2}, p\right) \cdots C\left(a_{s}, q\right)
$$

where $C(a, l)$ is defined by $(7)$ and $l, m, \ldots, q \in \mathbf{Z}_{+}^{n}$.

Lemma 5. If $a>-1, m=\left(m_{1}, \ldots, m_{n}\right) \in \mathbf{Z}_{+}^{n}, p=\left(p_{1}, \ldots, p_{n}\right) \in \mathbf{Z}_{+}^{n}, q=$ $\left(q_{1}, \ldots, q_{n}\right) \in \mathbf{Z}_{+}^{n}$ and $m=p+q, p_{j} q_{j}=0$ for $j=1,2, \ldots, n$, then

$$
C(a, m)=C(a, p) C(a+\sigma(p), q)
$$

where $C(a, l)$ is defined by (8).

Consider the special case that

$$
\begin{gathered}
p=m(j)=\left(m_{1}, \ldots, m_{j-1}, 0, m_{j+1}, \ldots, m_{n}\right), \\
q=m_{j} e_{j}=\left(0, \ldots, 0, m_{j}, 0, \ldots, 0\right) .
\end{gathered}
$$

Then (11) becomes

$$
C(a, m)=C(a, m(j)) C\left(a+\sigma(m(j)), m_{j} e_{j}\right)=C(a, m(j)) b\left(a+\sigma(m(j)), m_{j}\right)
$$

since

$$
C\left(a+\sigma(m(j)), m_{j} e_{j}\right)=b\left(a+\sigma(m(j)), m_{j}\right)
$$


If we take $p=m_{j} e_{j}, q=m(j)$, then (11) becomes

$$
C(a, m)=b\left(a, m_{j}\right) C\left(a+m_{j}, m(j)\right) .
$$

Thus we have

$$
C(a, m)=C(a, m(j)) b\left(a+\sigma(m(j)), m_{j}\right)=C\left(a+m_{j}, m(j)\right) b\left(a, m_{j}\right) .
$$

In particular, if $j=n$, then (12) becomes

$$
C(a, m)=C(a, m(n)) b\left(a+\sigma(m(n)), m_{n}\right)=C\left(a+m_{n}, m(n)\right) b\left(a, m_{n}\right) .
$$

Lemma 6. If $a, m$ are two real numbers, $m \geq 1$, and $m+a>0$, then there exist two positive numbers $c$ and $C$, which are independent of $m$, and only depend on $a$, such that

$$
c \Gamma(m) m^{a}<\Gamma(m+a)<C \Gamma(m) m^{a} .
$$

Proof. When $m$ is a positive integer, then (14) is just the consequence of EulerGauss formula

$$
\Gamma(a)=\lim _{m \rightarrow \infty} m^{a} \frac{1 \cdot 2 \cdots(m-1)}{a \cdot(a+1) \cdots(a+m)} .
$$

When $m$ is not a positive integer, we may prove it by using the Stirling's formula: For real $x>0$,

$$
\Gamma(x)=\sqrt{2 \pi} x^{x-1 / 2} e^{-x} e^{\theta(x) / 12 x}
$$

with $0<\theta(x)<1$. Using the Stirling formula, we have

$$
\frac{\Gamma(m+a)}{\Gamma(m) m^{a}}=\left(1+\frac{a}{m}\right)^{m+a-1 / 2} e^{-a} \exp \left\{\frac{\theta_{1}(m+a)}{12(m+a)}-\frac{\theta_{2}(m)}{12 m}\right\}
$$

where $0<\theta_{1}<1,0<\theta_{2}<1$. It is easy to verify that the right hand side of the previous equality is bounded above by $\exp \left\{1-a+\frac{1}{12(1+a)}\right\} \max \left[1,(1+a)^{a-1 / 2}\right]$, and bounded below by $\exp \left\{-\frac{1}{12}-a\right\} \min \left[1, e^{a}\right] \min \left[1,(1+a)^{a-1 / 2}\right]$. Thus we prove (14).

Actually, we have $\lim _{m \rightarrow \infty} \frac{\Gamma(m+a)}{\Gamma(m) m^{a}}=1$, for any positive real number sequence $\{m\}$.

\section{The Main Lemma}

Lemma 7 (Main Lemma). Let $u=\left(u_{1}, \ldots, u_{n}\right) \in \mathbf{R}_{+}^{n}, \sigma(u)<1, a>0$, $\varepsilon=$ $\left(\varepsilon_{1}, \varepsilon_{2}, \ldots, \varepsilon_{n}\right) \in \mathbf{R}^{n}, \varepsilon_{j}>-1, j=1,2, \ldots, n$. Further, if all $\varepsilon_{j}, j=1,2, \ldots, n$, are non-positive, we assume that $a+\sum_{j=1}^{n} \varepsilon_{j}>1$. If some of the $\varepsilon_{j}, j=1,2, \ldots, n$, are non-negative and the others are non-positive, then we assume that $a+\sum_{j=1}^{n} \varepsilon_{j}>$ $\sum_{j=1}^{n}\left\{\varepsilon_{j}\right\}+1$, where $\left\{\varepsilon_{j}\right\}$ means the least integer which is equal to or greater than $\varepsilon_{j}$. Let

$$
F_{n}^{a, \varepsilon}(u)=(1-\sigma(u))^{-a} \prod_{j=1}^{n}\left(1+u_{j}-\sigma(u)\right)^{-\varepsilon_{j}},
$$

and let the Taylor expansion of $F_{n}^{a, \varepsilon}(u)$ be

$$
F_{n}^{a, \varepsilon}(u)=\sum_{s \in \mathbf{Z}_{+}^{n}} B_{s, n}^{a, \varepsilon} u^{s}
$$


where $s=\left(s_{1}, s_{2}, \ldots, s_{n}\right) \in \mathbf{Z}_{+}^{n}, u^{s}=u_{1}^{s_{1}} \cdots u_{n}^{s_{n}}$, then there exist two positive constants $m_{n}$ and $M_{n}$, which are independent of $s$, and depend on $a, n$, and $\varepsilon$ only, such that

$$
m_{n} A_{s, n}^{a, \varepsilon} \leq B_{s, n}^{a, \varepsilon} \leq M_{n} A_{s, n}^{a, \varepsilon}
$$

where

$$
A_{s, n}^{a, \varepsilon}=\frac{\Gamma(a+\sigma(\varepsilon)+\sigma(s))}{\Gamma(a+\sigma(\varepsilon)) \prod_{j=1}^{n} \Gamma\left(1+s_{j}\right)\left(1+s_{j}\right)^{\varepsilon_{j}}}=\frac{C(a+\sigma(\varepsilon), s)}{\prod_{j=1}^{n}\left(1+s_{j}\right)^{\varepsilon_{j}}},
$$

and $\sigma(\varepsilon)=\sum_{j=1}^{n} \varepsilon_{j}, \sigma(s)=\sum_{j=1}^{n} s_{j}$.

We prove the Main Lemma by induction for $n$.

When $n=1$,

$$
F_{1}^{a, \varepsilon}(u)=\left(1-u_{1}\right)^{-a}=\sum b\left(a, m_{1}\right) u_{1}^{m_{1}},
$$

that is, $B_{m, 1}^{a, \varepsilon}=b\left(a, m_{1}\right),\left(m=m_{1}\right)$; and

$$
A_{m, 1}^{a, \varepsilon}=\frac{\Gamma\left(a+\varepsilon_{1}+m_{1}\right)}{\Gamma\left(a+\varepsilon_{1}\right) \Gamma\left(1+m_{1}\right)\left(1+m_{1}\right)^{\varepsilon_{1}}}=b\left(a, m_{1}\right) I_{1}
$$

where

$$
I_{1}=\frac{\Gamma\left(a+\varepsilon_{1}+m_{1}\right) \Gamma(a)}{\Gamma\left(a+\varepsilon_{1}\right) \Gamma\left(a+m_{1}\right)\left(1+m_{1}\right)^{\varepsilon_{1}}} .
$$

Obviously (17) is true when $m_{1}=0$. If $m_{1} \neq 0$, then by Lemma 6 , there exist $c$ and $C$, which depends only on $a$ and $\varepsilon$, such that

$$
I_{1} \leq C \frac{\Gamma(a)}{\Gamma\left(a+\varepsilon_{1}\right)}\left(\frac{m_{1}}{1+m_{1}}\right)^{\varepsilon_{1}}<C \frac{\Gamma(a)}{\Gamma\left(a+\varepsilon_{1}\right)}
$$

if $\varepsilon_{1} \geq 0 ;$ and

$$
I_{1} \leq C 2^{-\varepsilon_{1}} \frac{\Gamma(a)}{\Gamma\left(a+\varepsilon_{1}\right)}
$$

if $\varepsilon_{1}<0$. Similarly,

$$
I_{1}>c \frac{\Gamma(a)}{\Gamma\left(a+\varepsilon_{1}\right)}
$$

if $\varepsilon_{1}<0$; and

$$
I_{1}>c 2^{-\varepsilon_{1}} \frac{\Gamma(a)}{\Gamma\left(a+\varepsilon_{1}\right)}
$$

if $\varepsilon_{1} \geq 0$. We have proved (17) in the case $n=1$.

In order to complete the induction process, we will need the following lemmas.

Lemma 8. The assumptions are as in the Main Lemma. Moreover, we assume for a fixed $k, 1 \leq k \leq n, \varepsilon_{k}>0$, then

$$
B_{s, n}^{a, \varepsilon}=\sum_{l(k)+\eta(k)=s(k)} C\left(\varepsilon_{k}, \eta(k)\right) b\left(a+\sigma(\varepsilon(k))+\sigma(l(k)), s_{k}\right) B_{l(k), n-1}^{a, \varepsilon(k)} ;
$$

if we assume $\varepsilon_{k}=0$, then

$$
\left.B_{s, n}^{a, \varepsilon}=b(a+\sigma(\varepsilon))+\sigma(s(k)), s_{k}\right) B_{s(k), n-1}^{a, \varepsilon(k)} ;
$$


where $l=\left(l_{1}, \ldots, l_{n}\right) \in \mathbf{Z}_{+}^{n}, \eta=\left(\eta_{1}, \ldots, \eta_{n}\right) \in \mathbf{Z}_{+}^{n},\left(s_{1}, \ldots, s_{n}\right) \in \mathbf{Z}_{+}^{n}$ and $B_{s(k), n-1}^{a, \varepsilon(k)}$ is the coefficient of the Taylor expansion of the function

$$
F_{n-1}^{a, \varepsilon(k)}(u(k))=\left(1-\sigma(u(k))^{-a} \prod_{j \neq k}\left(1-\sigma(u(k))+u_{j}\right)^{-\varepsilon_{j}} .\right.
$$

Lemma 9. The assumptions are as in the Main Lemma. Fix any $k, 1 \leq k \leq n$, then

$$
\begin{array}{rl}
B_{a, n}^{a, \varepsilon}=\sum_{l+\xi=s} & b\left(a_{k}, \xi_{k}\right) C\left(a_{k}+\varepsilon_{k}+\xi_{k}, \xi(k)\right) \\
& \times b\left(a-a_{k}+\sigma(\varepsilon(k))+\sigma(l(k)), l_{k}\right) B_{l(k), n-1}^{a-a_{k}, \varepsilon(k)}
\end{array}
$$

where $a=a_{1}+a_{2}+\cdots+a_{n}$, such that $a_{j}>0, a_{j}+\varepsilon_{j}>0, j=1,2, \ldots, n$, and $l=\left(l_{1}, l_{2}, \ldots, l_{n}\right) \in \mathbf{Z}_{+}^{n}, \xi=\left(\xi_{1}, \xi_{2}, \ldots, \xi_{n}\right) \in \mathbf{Z}_{+}^{n} ; s=\left(s_{1}, s_{2}, \ldots, s_{n}\right) \in \mathbf{Z}_{+}^{n}$ and $B_{l(k), n-1}^{a-a_{k}, \varepsilon(k)}$ is the coefficient of the Taylor expansion of the function

$$
F_{n-1}^{a-a_{k}, \varepsilon(k)}(u(k))=(1-\sigma(u(k)))^{a_{k}-a} \prod_{j \neq k}\left(1-\sigma(u(k))+u_{j}\right)^{-\varepsilon_{j}} .
$$

Lemma 10. Assumptions are as in the Main Lemma, then all the coefficients $B_{s, n}^{a, \varepsilon}$ in (16) are non-negative.

Proof of Lemma 8. In the case $\varepsilon_{k}>0$, we can expand each factor in (15) by power series, then

$$
\begin{aligned}
F_{n}^{a, \varepsilon}(u) & =\sum_{m, \beta(1), \ldots, \gamma(n-1), \delta(n) \in \mathbf{Z}_{+}^{n}} C(a, m) C\left(\varepsilon_{1}, \beta(1)\right) \cdots \\
& =\sum_{s \in \mathbf{Z}_{+}^{n} m+\beta(1)+\cdots+\gamma(n-1)+\delta(n)=s} C\left(\varepsilon_{n-1}, \gamma(n-1)\right) C\left(\varepsilon_{n}, \delta(n)\right) u^{m+\beta(1)+\cdots+\delta(n)} \\
& C(a, m) C\left(\varepsilon_{1}, \beta(1)\right) \cdots C\left(\varepsilon_{n}, \delta(n)\right) u^{s} .
\end{aligned}
$$

Comparing with (16), we get

$$
B_{s, n}^{a, \varepsilon}=\sum_{m+\beta(1)+\cdots+\delta(n)=s} C(a, m) C\left(\varepsilon_{1}, \beta(1)\right) \cdots C\left(\varepsilon_{n}, \delta(n)\right) .
$$

Using Lemma 5, we can decompose each factor (except the factor $\left.C\left(\varepsilon_{k}, \xi(k)\right)\right)$ on the right-hand side of this equality as

$$
\begin{gathered}
C(a, m)=C(a, m(k)) b\left(a+\sigma(m(k)), m_{k}\right), \\
C\left(\varepsilon_{1}, \beta(1)\right)=C\left(\varepsilon_{1}, \beta(1, k)\right) b\left(\varepsilon_{1}+\sigma(\beta(1, k)), \beta_{k}\right), \\
\ldots \ldots \\
C\left(\varepsilon_{n}, \delta(n)\right)=C\left(\varepsilon_{n}, \delta(k, n)\right) b\left(\varepsilon_{n}+\sigma(\delta(k, n)), \delta_{k}\right) .
\end{gathered}
$$


Then we have

$$
\begin{aligned}
B_{s, n}^{a, \varepsilon}= & \sum_{m+\beta(1)+\cdots+\delta(n)=s} C\left(\varepsilon_{k}, \xi(k)\right) C(a, m(k)) C\left(\varepsilon_{1}, \beta(1, k)\right) \cdots \\
& {\left[C\left(\varepsilon_{k}, \xi(k, k)\right)\right] \cdots C\left(\varepsilon_{n}, \delta(k, n)\right) b\left(a+\sigma(m(k)), m_{k}\right) } \\
& \times b\left(\varepsilon_{1}+\sigma(\beta(1, k)), \beta_{k}\right) \cdots\left[b\left(\varepsilon_{k}+\sigma(\xi(k, k)), \beta_{k}\right)\right] \cdots b\left(\varepsilon_{n}+\sigma(\delta(k, n)), \delta_{n}\right) \\
= & \sum_{l(k)+\xi(k)=s(k)} C\left(\varepsilon_{k}, \xi(k)\right) \sum_{m(k)+\beta(1, k)+\cdots+\delta(k, n)=l(k)} C(a, m(k)) \\
& \times C\left(\varepsilon_{1}, \beta(1, k)\right) \cdots\left[C\left(\varepsilon_{k}, \xi(k, k)\right)\right] \cdots C\left(\varepsilon_{n}, \delta(k, n)\right) \\
& \times \sum_{m_{k}+\beta_{k}+\cdots+\delta_{k}=s_{k}} b\left(a+\sigma(m(k)), m_{k}\right) b\left(\varepsilon_{1}+\sigma(\beta(1, k)), \beta_{k}\right) \cdots \\
& {\left[b\left(\varepsilon_{k}+\sigma(\xi(k, k)), \beta_{k}\right)\right] \cdots b\left(\varepsilon_{n}+\sigma(\delta(k, n)), \delta_{n}\right) . }
\end{aligned}
$$

where [ ] means that the term inside [] is deleted from the product. By Lemma 3, the last sum of the right hand side of this equality is equal to $b\left(a+\sigma(\varepsilon(k))+\sigma(l(k)), s_{k}\right)$, and the second sum is just the coefficient $B_{l(k), n-1}^{a, \varepsilon(k)}$ of the Taylor expansion of the function (21).

In the case $\varepsilon_{k}=0$, we have

$$
B_{s, n}^{a, \varepsilon}=\sum_{m+\beta(1)+\cdots+\delta(n)=s} C(a, m) C\left(\varepsilon_{1}, \beta(1)\right) \cdots\left[C\left(\varepsilon_{k}, \xi(k)\right)\right] \cdots C\left(\varepsilon_{n}, \delta(n)\right),
$$

and

$$
\begin{aligned}
B_{s, n}^{a, \varepsilon}= & \sum_{\begin{array}{l}
m(k)+\beta(1, k)+\cdots+\delta(k, n)=l(k) \\
\end{array}} C(a, m(k)) C\left(\varepsilon_{1}, \beta(1, k)\right) \cdots \\
& \left.\times \sum_{\substack{m_{k}+\beta_{k}+\cdots+\delta_{k}=s_{k}\\
}} b(a+\sigma(k))\right] \cdots C\left(\varepsilon_{n}, \delta(k, n)\right) \\
& {\left[b\left(\varepsilon_{k}+\sigma(\xi(k, k)), \xi_{k}\right)\right] \cdots b\left(\varepsilon_{n}+\sigma(\delta(k, n)), m_{k}\right) b\left(\varepsilon_{1}+\sigma(\beta(1, k)), \beta_{k}\right) \cdots }
\end{aligned}
$$

We get (20).

We have proved Lemma 8.

Proof of Lemma 9. We can express $F_{n}^{a, \varepsilon}(u)$ as

$$
F_{n}^{a, \varepsilon}=\prod_{j=1}^{n}\left[(1-\sigma(u))^{-a_{j}}(1-\sigma(u(j)))^{-\varepsilon_{j}}\right] .
$$

Since $\sigma(u)=\sigma(u(j))+u_{j}$, we get

$$
\begin{aligned}
(1 & -\sigma(u))^{-a_{j}}(1-\sigma(u(j)))^{-\varepsilon_{j}} \\
& =\left(1-u_{j}\left(1-\sigma(u(j))^{-1}\right)^{-a_{j}}(1-\sigma(u(j)))^{-\varepsilon_{j}-a_{j}} .\right.
\end{aligned}
$$

The assumption $\sigma(u)<1$ implies $u_{j}(1-\sigma(u(j)))^{-1}<1$,

$$
\left(1-u_{j}(1-\sigma(u(j)))^{-1}\right)^{-a_{j}}=\sum_{k=0}^{\infty} b\left(a_{j}, k\right) u_{j}^{k}(1-\sigma(u(j)))^{-k}
$$


and

$$
\begin{aligned}
(1- & \sigma(u))^{-a_{j}}(1-\sigma(u(j)))^{-\varepsilon_{j}}=\sum_{k=0}^{\infty} b\left(a_{j}, k\right) u_{j}^{k}(1-\sigma(u(j)))^{-\varepsilon_{j}-a_{j}-k} \\
& =\sum_{k=0}^{\infty} b\left(a_{j}, k\right) u_{j}^{k} \sum_{m(j) \in \mathbf{Z}_{+}^{n}} C\left(\varepsilon_{j}+a_{j}+k, m(j)\right) u^{m(j)} \\
& =\sum_{m \in \mathbf{Z}_{+}^{n}} b\left(a_{j}, m_{j}\right) C\left(a_{j}+\varepsilon_{j}+m_{j}, m(j)\right) u^{m}
\end{aligned}
$$

where $\sum_{m(j) \in \mathbf{Z}_{+}^{n}}$ means the sum taking for all lattice points in $\mathbf{Z}_{+}^{n}$, where the $j$ th coordinate is zero.

Thus we can expand every factor in (23) by (24) as

$$
\begin{aligned}
F_{n}^{a, \varepsilon}(u) & =\sum_{\beta, \gamma, \ldots, \delta, \eta \in \mathbf{Z}_{+}^{n}} b\left(a_{1}, \beta_{1}\right) C\left(a_{1}+\varepsilon_{1}+\beta_{1}, \beta(1)\right) \cdots \\
& b\left(a_{n}, \eta_{n}\right) C\left(a_{n}+\varepsilon_{n}+\eta_{n}, \eta(n)\right) u^{\beta+\gamma+\cdots+\delta+\eta} \\
& =\sum_{s \in \mathbf{Z}_{+}^{n}} \sum_{\beta+\gamma+\cdots+\delta+\eta=s} b\left(a_{1}, \beta_{1}\right) C\left(a_{1}+\varepsilon_{1}+\beta_{1}, \beta(1)\right) \cdots \\
& b\left(a_{n}, \eta_{n}\right) C\left(a_{n}+\varepsilon_{n}+\eta_{n}, \eta(n)\right) u^{s} .
\end{aligned}
$$

Comparing this formula with (16), we get

$$
\begin{gathered}
B_{s, n}^{a, \varepsilon}=\sum_{\substack{\beta+\gamma+\cdots+\delta+\eta=s \\
b\left(a_{n}, \eta_{n}\right) C\left(a_{n}+\varepsilon_{n}+\eta_{n}, \eta(n)\right) .}} b\left(a_{1}, \beta_{1}\right) C\left(a_{1}+\varepsilon_{1}+\beta_{1}, \beta(1)\right) \cdots
\end{gathered}
$$

Let

$$
\begin{aligned}
& \beta(1, k)=\left(0, \beta_{2}, \ldots, \beta_{k-1}, 0, \beta_{k+1}, \ldots, \beta_{n}\right), \\
& \gamma(2, k)=\left(\gamma_{1}, 0, \gamma_{3}, \ldots, \gamma_{k-1}, 0, \gamma_{k+1}, \ldots, \gamma_{n}\right),
\end{aligned}
$$

etc., then we may decompose $C\left(a_{1}+\varepsilon_{1}+\beta_{1}, \beta(1)\right)$, etc., by Lemma 5 as,

$$
\begin{aligned}
C\left(a_{1}+\varepsilon_{1}+\beta_{1}, \beta(1)\right) & =C\left(a_{1}+\varepsilon_{1}+\beta_{1}, \beta(1, k)\right) b\left(a_{1}+\varepsilon_{1}+\sigma(\beta(k)), \beta_{k}\right), \\
C\left(a_{2}+\varepsilon_{2}+\gamma_{2}, \gamma(2)\right) & =C\left(a_{2}+\varepsilon_{2}+\gamma(2, k)\right) b\left(a_{2}+\varepsilon_{2}+\sigma(\gamma(k)), \gamma_{k}\right), \\
& \ldots \ldots \ldots \\
C\left(a_{n}+\varepsilon_{n}+\eta_{n}, \eta(n)\right) & =C\left(a_{n}+\varepsilon+\eta_{n}, \eta(k, n)\right) b\left(a_{n}+\varepsilon+\sigma(\eta(k)), \eta_{k}\right)
\end{aligned}
$$

except the term $C\left(a_{k}+\varepsilon_{k}+\xi_{k}, \xi(k)\right)$. 
Substituting the above equalities into (25), and let $l=s-\xi$, we get

$$
\begin{aligned}
B_{s, n}^{a, \varepsilon}= & \sum_{l+\xi=s} b\left(a_{k}, \xi_{k}\right) C\left(a_{k}+\varepsilon_{k}+\xi_{k}, \xi(k)\right) \sum_{\beta+\gamma+\cdots+\eta=l} b\left(a_{1}, \beta_{1}\right) \\
& \times C\left(a_{1}+\varepsilon_{1}+\beta_{1}, \beta(1, k)\right) b\left(a_{2}, \gamma_{2}\right) C\left(a_{2}+\varepsilon_{2}+\gamma_{2}, \gamma(2, k)\right) \cdots \\
& {\left[b\left(a_{k}, \xi_{k}\right) C\left(a_{k}+\varepsilon_{k}+\xi_{k}, \xi(k, k)\right)\right] \cdots b\left(a_{n}, \eta_{n}\right) C\left(a_{n}+\varepsilon_{n}+\eta_{n}, \eta(k, n)\right) } \\
& \times b\left(a_{1}+\varepsilon_{1}+\sigma(\beta(k)), \beta_{k}\right) b\left(a_{2}+\varepsilon+\sigma(\gamma(k)), \gamma_{k}\right) \\
& \cdots\left[b\left(a_{k}+\varepsilon_{k}+\sigma(\xi(k)), \xi_{k}\right)\right] \cdots b\left(a_{n}+\varepsilon_{n}+\sigma(\eta(k)), \eta_{k}\right) \\
= & \sum_{l+\xi=s} b\left(a_{k}, \xi_{k}\right) C\left(a_{k}+\varepsilon_{k}+\xi_{k}, \xi(k)\right) b\left(a-a_{k}+\sigma(\varepsilon(k))+\sigma(l(k)), l_{k}\right) \\
& \times \sum_{\beta(k)+\gamma(k)+\cdots+\eta(k)=l(k)} b\left(a_{1}, \beta_{1}\right) C\left(a_{1}+\varepsilon+\beta_{1}, \beta(1, k)\right) \cdots \\
& {\left[b\left(a_{k}, \xi_{k}\right) C\left(a_{k}+\varepsilon_{k}+\xi_{k}, \xi(k, k)\right)\right] \cdots b\left(a_{k}, \eta_{k}\right) C\left(a_{n}+\varepsilon_{n}+\eta_{n}, \eta(k, n)\right) }
\end{aligned}
$$

by Lemma 3, where [ ] means that the term inside [ ] is deleted from the product. The last sum of the right-hand side of the previous equality is just the coefficient $B_{l(k), n-1}^{a-a_{k}, \varepsilon(k)}$ of the Taylor expansion of the function

$$
\begin{aligned}
F_{n-1}^{a-a_{k}, \varepsilon(k)} & =(1-\sigma(u(k)))^{a_{k}-a} \prod_{j \neq k}\left(1+u_{j}-\sigma(u(k))\right)^{-\varepsilon_{j}} \\
& =\sum_{l(k) \in \mathbf{Z}_{+}^{n}} B_{l(k), n-1}^{a-a_{k}, \varepsilon(k)} u^{l(k)} .
\end{aligned}
$$

We have proved Lemma 9.

Proof of Lemma 10. By the assumptions of the Main Lemma, we may choose $a_{j}>$ $0, j=1,2, \ldots, n$, such that $a=a_{1}+\cdots+a_{n}$, and $a_{j}+\varepsilon_{j}>0, j=1,2, \ldots, n$, then $B_{s, n}^{a, \varepsilon} \geq 0$ for all $s$ follows by (25).

\section{Proof of the Main Lemma, Part 1}

Fix $s=\left(s_{1}, s_{2}, \ldots, s_{n}\right) \in \mathbf{Z}_{+}^{n}$, and let $s_{k}=\max _{1 \leq j \leq n} s_{j}$. We may assume $s_{k} \geq 1$, otherwise $s=0$, then the Main Lemma is true obviously. For that $k$, there are three possibilities of $\varepsilon_{k}$ : (1) $\varepsilon_{i}>0 ;(2) \varepsilon_{k}=0$ and $(3) \varepsilon_{k}<0$.

In this section, we prove the Main Lemma in the cases (1) $\varepsilon_{k}>0$ holds or (2) $\varepsilon_{k}=0$ holds.

If $\varepsilon_{k}>0$, by the conditions of the Main Lemma, all the factors in the right-hand side of (19) are non-negative.

The induction process is as follows: to prove (17) is true if we assume

$$
m_{n-1}(j) A_{s(j), n-1}^{a, \varepsilon(j)} \leq B_{s(j), n-1}^{a, \varepsilon(j)} \leq M_{n-1}(j) A_{s(j), n-1}^{a, \varepsilon(j)}
$$

is true for $j=1,2, \ldots, n$ where $m_{n-1}(j)$ and $M_{n-1}(j)$ are two positive constants which are independent of $s(j)$, and only dependent on $a$ and $\varepsilon$, and

$$
A_{l(j), n-1}^{a, \varepsilon(j)}=C(a+\sigma(\varepsilon(j)), l(j)) \prod_{p \neq j}\left(1+l_{p}\right)^{-\varepsilon_{p}} .
$$

Let $m_{n-1}=\min _{1 \leq j \leq n} m_{n-1}(j)$ and $M_{n-1}=\max _{1 \leq j \leq n} M_{n-1}(j)$, then

$$
m_{n-1} A_{l(j), n-1}^{a, \varepsilon(j)} \leq B_{l(j), n-1}^{a, \varepsilon(k)} \leq M_{n-1} A_{l(j), n-1}^{a, \varepsilon(j)} .
$$


In particular, if we take $j=k$, we have

$$
m_{n-1} A_{l(k), n-1}^{a, \varepsilon(k)} \leq B_{l(k), n-1}^{a, \varepsilon(k)} \leq M_{n-1} A_{l(k), n-1}^{a, \varepsilon(k)},
$$

where

$$
A_{l(k), n-1}^{a, \varepsilon(k)}=C(a+\sigma(\varepsilon(k)), l(k)) \prod_{j \neq k}\left(1+l_{j}\right)^{-\varepsilon_{j}} .
$$

Let

$$
I_{s}^{a, \varepsilon}=\sum_{l(k)+\eta(k)=s(k)} C\left(\varepsilon_{k}, \eta(k)\right) b\left(a+\sigma(\varepsilon(k))+\sigma(l(k)), s_{k}\right) A_{l(k), n-1}^{a, \varepsilon(k)}
$$

then

$$
m_{n-1} I_{s}^{a, \varepsilon} \leq B_{s, n}^{a, \varepsilon} \leq M_{n-1} I_{s}^{a, \varepsilon}
$$

by (19).

We estimate $I_{s}^{a, \varepsilon}$

Fix $l_{k}$. We define the set $E$ as

$$
E=\left\{l(k)=\left(l_{1}, \ldots, l_{k-1}, 0, l_{k+1}, \ldots, l_{n}\right) \in \mathbf{Z}_{+}^{n}, 0 \leq l_{j} \leq s_{j}, j \neq k\right\} .
$$

We define the mapping $\phi: E \rightarrow E$ as

$$
\phi(l(k))=\bar{l}(k)=\left(\bar{l}_{1}, \ldots, \bar{l}_{k-1}, 0, \bar{l}_{k+1}, \ldots, \bar{l}_{n}\right)
$$

where $\bar{l}_{j}=l_{j}$, if $\frac{1}{2} s_{j} \leq l_{j} \leq s_{j} ; \bar{l}_{j}=s_{j}-l_{j}$, if $0 \leq l_{j}<\frac{1}{2} s_{j}$; and define $\bar{\eta}(k)$ as $\bar{l}(k)+\bar{\eta}(k)=l(k)+\eta(k)=s(k)$.

It is easily verified that

$$
\prod_{j \neq k} \Gamma\left(1+\bar{\eta}_{j}\right) \Gamma\left(1+\bar{l}_{j}\right)=\prod_{j \neq k} \Gamma\left(1+\eta_{j}\right) \Gamma\left(1+l_{j}\right) .
$$

Consider

$$
I \equiv \frac{C\left(\varepsilon_{k}, \bar{\eta}(k)\right) b\left(a+\sigma(\varepsilon(k))+\sigma(\bar{l}(k)), s_{k}\right) A_{\bar{l}(k), n-1}^{a, \varepsilon(k)}}{C\left(\varepsilon_{k}, \eta(k)\right) b\left(a+\sigma(\varepsilon(k))+\sigma(l(k)), s_{k}\right) A_{l(k), n-1}^{a, \varepsilon(k)}} .
$$

By (26) and (27), we have

$$
I=\frac{\Gamma\left(\varepsilon_{k}+\sigma(\bar{\eta}(k))\right) \Gamma\left(a+\sigma(\varepsilon(k))+\sigma(\bar{l}(k))+s_{k}\right)}{\Gamma\left(\varepsilon_{k}+\sigma(\eta(k))\right) \Gamma\left(a+\sigma(\varepsilon(k))+\sigma(l(k))+s_{k}\right)} \prod_{j \neq k}\left(\frac{1+l_{j}}{1+\bar{l}_{j}}\right)^{\varepsilon_{j}} .
$$

If all $\bar{l}_{j}=l_{j}, j \neq k$, then $I=1$. If at least one of $\bar{l}_{j} \neq l_{j}$, then $d=\sigma(\bar{l}(k))-\sigma(l(k))=$ $\sigma(\eta(k))-\sigma(\bar{\eta}(k)) \geq 1$. Let $d_{j}=\bar{l}_{j}-l_{j}, j \neq k$, then

$$
I=\prod_{\nu=0}^{d-1} \frac{a+\sigma(\varepsilon(k))+s_{k}+\sigma(l(k))+\nu}{\varepsilon_{k}+\sigma(\bar{\eta}(k))+\nu} \prod_{j \neq k}\left(\frac{1+l_{j}}{1+l_{j}+d_{j}}\right)^{\varepsilon_{j}} .
$$


The reciprocal of the first product in the previous equality is

$$
\begin{aligned}
\prod_{\nu=0}^{d-1} & \frac{\varepsilon_{k}+\sigma(\bar{\eta}(k))+\nu}{a+\sigma(\varepsilon(k))+s_{k}+\sigma(l(k))+\nu} \\
& =\prod_{\nu=0}^{d-1}\left(1+\frac{\varepsilon_{k}+\sigma(\bar{\eta}(k))-a-\sigma(\varepsilon(k))-s_{k}-\sigma(l(k))}{a+\sigma(\varepsilon(k))+s_{k}+\sigma(l(k))+\nu}\right) \\
& \leq \prod_{\nu=0}^{d-1} \exp \left\{\frac{\varepsilon_{k}+\sigma(\bar{\eta}(k))-a-\sigma(\varepsilon(k))-s_{k}-\sigma(l(k))}{a+\sigma(\varepsilon(k))+s_{k}+\sigma(l(k))+\nu}\right\}
\end{aligned}
$$

since $e^{x} \geq 1+x$ when $x \geq-1$. Then

$$
I \geq \exp \left(\sum_{\nu=0}^{d-1} \frac{a+\sigma(\varepsilon(k))+s_{k}+\sigma(l(k))-\sigma(\bar{\eta}(k))-\varepsilon_{k}}{a+\sigma(\varepsilon(k))+s_{k}+\sigma(l(k))+\nu}\right) \prod_{j \neq k}\left(\frac{1+l_{j}}{1+l_{j}+d_{j}}\right)^{\varepsilon_{j}} .
$$

When $\varepsilon$ is given and $s_{k}=\max _{1 \leq j \leq n} s_{j}$, there are only a finite number of $s \in \mathbf{Z}_{+}^{n}$ such that $s_{k} \leq 2 \varepsilon_{k}$. In this situation, we only have a finite number of $B_{s, n}^{a, \varepsilon}$ and $A_{s, n}^{a, \varepsilon}$, thus we just need to take

$$
M_{n}=\max _{s_{k}=\max s_{j}, s_{k} \leq 2 \varepsilon_{k}} \frac{B_{s, n}^{a, \varepsilon}}{A_{s, n}^{a, \varepsilon}}, \quad m_{n}=\min _{s_{k}=\max s_{j}, s_{k} \leq 2 \varepsilon_{k}} \frac{B_{s, n}^{a, \varepsilon}}{A_{s, n}^{a, \varepsilon}} .
$$

It only remains for us to consider the case $s_{k} \geq 2 \varepsilon_{k}$.

From the definition of the mapping $\phi$, we know $\bar{\eta}_{j} \leq l_{j}, j \neq k$, and hence $\sigma(l(k)) \geq \sigma(\bar{\eta}(k))$. Then

$$
\begin{aligned}
& \frac{a+\sigma(\varepsilon(k))+s_{k}+\sigma(l(k))-\sigma(\bar{\eta}(k))-\varepsilon_{k}}{a+\sigma(\varepsilon(k))+s_{k}+\sigma(l(k))+\nu} \\
& \quad>\frac{s_{k}+\sigma(l(k))-\sigma(\bar{\eta}(k))-\varepsilon_{k}}{s_{k}+\sigma(l(k))+\nu}>\frac{\frac{1}{2} s_{k}}{s_{k}+\sigma(l(k))+\nu}
\end{aligned}
$$

since $s_{k} \geq 2 \varepsilon_{k}$. We have

$$
I>\exp \left\{\sum_{\nu=0}^{d-1} \frac{s_{k}}{2\left(s_{k}+\sigma(l(k))+\nu\right)}\right\} \prod_{j \neq k}\left(\frac{1+l_{j}}{1+l_{j}+d_{j}}\right)^{\varepsilon_{j}},
$$

thus

$$
I>\exp \left\{\sum_{\nu=0}^{d-1} \frac{s_{k}}{2\left(s_{k}+\sigma(l(k))+\nu\right)}\right\} \prod_{j \neq k}\left(\frac{1}{1+d_{j}}\right)^{\left|\varepsilon_{j}\right|}
$$

since

$$
\left(\frac{1+l_{j}}{1+l_{j}+d_{j}}\right)^{\varepsilon_{j}} \geq\left(\frac{1}{1+d_{j}}\right)^{\left|\varepsilon_{j}\right|}
$$

Moreover, since $0 \leq \sigma(l(k)) \leq \sigma(s(k)) \leq(n-1) s_{k}$,

$$
\nu \leq d \leq \sigma(\bar{l}(k))-\sigma(l(k)) \leq \sigma(\bar{l}(k)) \leq(s(k)) \leq(n-1) s_{k},
$$

we have

$$
\frac{s_{k}}{2\left(s_{k}+\sigma(l(k))+\nu\right)} \geq \frac{s_{k}}{2\left(s_{k}+(n-1) s_{k}+(n-1) s_{k}\right)}=\frac{1}{4 n-2} .
$$


Therefore, we obtain

$$
I>\left(\frac{1}{1+d}\right)^{\sum_{j \neq k}\left|\varepsilon_{j}\right|} \exp \left(\frac{d}{4 n-2}\right) \equiv f(d) .
$$

The function $f(d)$ takes the minimum value at $d=(4 n-2) \sum_{j \neq k}\left|\varepsilon_{j}\right|-1$, hence

$$
I>e^{-1}\left((4 n-2) \sum_{j \neq k}\left|\varepsilon_{j}\right|\right)^{-\sum_{j \neq k}\left|\varepsilon_{j}\right|} \equiv a_{0}
$$

where $a_{0}$ is a constant which only depends on $\varepsilon$ and $n$. From the definition of $I$, the following inequality

$$
\begin{aligned}
& C\left(\varepsilon_{k}, \eta(k)\right) b\left(a+\sigma(\varepsilon(k))+\sigma(l(k)), s_{k}\right) A_{l(k), n-1}^{a, \varepsilon(k)} \\
& \quad \leq a_{0}^{-1} C\left(\varepsilon_{k}, \bar{\eta}(k)\right) b\left(a+\sigma(\varepsilon(k))+\sigma(\bar{l}(k)), s_{k}\right) A_{\bar{l}(k), n-1}^{a, \varepsilon(k)}
\end{aligned}
$$

holds. According to the definition of the set $E$ and the mapping $\phi$, at most $2^{n-1}$ different $l(k)$ map to the same $\bar{l}(k)$ by $\phi$. Let $\bar{E}=\phi(E)$, and

$$
J=\sum_{l(k)+\eta(k)=s(k), l(k) \in \bar{E}} C\left(\varepsilon_{k}, \eta(k)\right) b\left(a+\sigma(\varepsilon(k))+\sigma(l(k)), s_{k}\right) A_{l(k), n-1}^{a, \varepsilon(k)},
$$

then

$$
J \leq I_{s, n}^{a, \varepsilon}
$$

and

$$
\begin{aligned}
I_{s, n}^{a, \varepsilon}= & \sum_{l(k)+\eta(k)=s(k)} C\left(\varepsilon_{k}, \eta(k)\right) b\left(a+\sigma(\varepsilon(k))+\sigma(l(k)), s_{k}\right) A_{l(k), n-1}^{a, \varepsilon(k)} \\
\leq & 2^{n-1} a_{0}^{-1} \sum_{l(k)+\eta(k)=s(k), l(k) \in \bar{E}} C\left(\varepsilon_{k}, \eta(k)\right) \\
& \times b\left(a+\sigma(\varepsilon(k))+\sigma(l(k)), s_{k}\right) A_{l(k), n-1}^{a, \varepsilon(k)} \\
= & 2^{n-1} a_{0}^{-1} J .
\end{aligned}
$$

Thus

$$
J \leq I_{s}^{a, \varepsilon} \leq 2^{n-1} a_{0}^{-1} J
$$

Hence, we only need to estimate $J$.

Since $l \in \bar{E}, \frac{1}{2} s_{j} \leq l_{j} \leq s_{j}, j \neq k$, we have

$$
2^{-\left|\varepsilon_{j}\right|}\left(1+s_{j}\right)^{-\varepsilon_{j}} \leq\left(1+l_{j}\right)^{-\varepsilon_{j}} \leq 2^{\left|\varepsilon_{j}\right|}\left(1+s_{j}\right)^{-\varepsilon_{j}}
$$


for $j \neq k$. By (26) and (12),

$$
\begin{gathered}
J=\sum_{\substack{l(k)+\eta(k)=s(k), l(k) \in \bar{E} \\
\times}} C(a+\sigma(\varepsilon(k)), l(k)) \prod_{j \neq k}\left(1+l_{j}\right)^{-\varepsilon_{j}} \\
=\sum_{\substack{l(k)+\eta(k)=s(k), l(k) \in \bar{E} \\
\times b\left(a+\sigma(\varepsilon(k)), s_{k}\right)}} C\left(\varepsilon_{k}, \eta(k)\right) C\left(a+\sigma(\varepsilon(k))+s_{k}, l(k)\right) \\
\quad\left(1+l_{j}\right)^{-\varepsilon_{j}} .
\end{gathered}
$$

By (28), we get $2^{-\sum_{j \neq k}\left|\varepsilon_{j}\right|} L \leq J \leq 2^{\sum_{j \neq k}\left|\varepsilon_{j}\right|} L$, where

$$
\begin{aligned}
L= & b\left(a+\sigma(\varepsilon(k)), s_{k}\right) \prod_{j \neq k}\left(1+s_{j}\right)^{-\varepsilon_{j}} \\
& \times \sum_{l(k)+\eta(k)=s(k), l(k) \in \bar{E}} C\left(\varepsilon_{k}, \eta(k)\right) C\left(a+\sigma(\varepsilon(k))+s_{k}, l(k)\right) .
\end{aligned}
$$

Using Lemma 4, we have

$$
L \leq \prod_{j \neq k}\left(1+s_{j}\right)^{-\varepsilon_{j}} b\left(a+\sigma(\varepsilon(k)), s_{k}\right) C\left(a+\sigma(\varepsilon)+s_{k}, s(k)\right)
$$

Using Lemma 6, we have

$$
\begin{aligned}
& \frac{b\left(a+\sigma(\varepsilon(k)), s_{k}\right)\left(1+s_{k}\right)^{\varepsilon_{k}}}{b\left(a+\sigma(\varepsilon), s_{k}\right)} \\
& \quad=\frac{\Gamma\left(a+\sigma(\varepsilon(k))+s_{k}\right) \Gamma\left(a+\sigma(\varepsilon(k))+\varepsilon_{k}\right)\left(1+s_{k}\right)^{\varepsilon_{k}}}{\Gamma(a+\sigma(\varepsilon(k))) \Gamma\left(a+\sigma(\varepsilon(k))+\varepsilon_{k}+s_{k}\right)} \\
& \quad \leq C \frac{\Gamma(a+\sigma(\varepsilon))}{\Gamma(a+\sigma(\varepsilon(k)))}\left(\frac{1+s_{k}}{a+\sigma(\varepsilon)+s_{k}}\right)^{\varepsilon_{k}} \\
& \quad \leq C \frac{\Gamma(a+\sigma(\varepsilon))}{\Gamma(a+\sigma(\varepsilon(k)))} \max \left(1,(a+\sigma(\varepsilon))^{-\varepsilon_{k}}\right)=C_{1} .
\end{aligned}
$$

Of course, the constant $C_{1}$ depends only on $a$ and $\varepsilon$. Thus

$$
\begin{array}{r}
J \leq 2^{\sum_{j \neq k}\left|\varepsilon_{j}\right|} C_{1} \prod_{j=1}^{n}\left(1+s_{j}\right)^{-\varepsilon_{j}} C\left(a+\sigma(\varepsilon)+s_{k}, s(k)\right) b\left(a+\sigma(\varepsilon), s_{k}\right) \\
=2^{\sum_{j \neq k}\left|\varepsilon_{j}\right|} C_{1} C(a+\sigma(\varepsilon), s) \prod_{j=1}^{n}\left(1+s_{j}\right)^{-\varepsilon_{j}}=2^{\sum_{j \neq k}\left|\varepsilon_{j}\right|} C_{1} A_{s, n}^{a, \varepsilon}
\end{array}
$$

by (12) and (18)

We have proved the right-hand inequality of (17) when $\varepsilon_{k}>0$.

Now we are going to prove the left-hand inequality of (17) when $\varepsilon_{k}>0$.

From the definition of $I$,

$$
I=I_{0} \prod_{j \neq k}\left(\frac{1+\bar{l}_{j}}{1+l_{j}}\right)^{-\varepsilon_{j}}
$$


where $I_{0}$ is equal to

$$
\frac{C\left(\varepsilon_{k}, \bar{\eta}(k)\right) b\left(a+\sigma(\varepsilon(k))+\sigma(\bar{l}(k)), s_{k}\right) C(a+\sigma(\varepsilon(k)), \bar{l}(k))}{C\left(\varepsilon_{k}, \eta(k)\right) b\left(a+\sigma(\varepsilon(k))+\sigma(l(k)), s_{k}\right) C(a+\sigma(\varepsilon(k)), l(k))} .
$$

Then

$$
\begin{aligned}
I_{0}= & \frac{\Gamma\left(\varepsilon_{k}+\sigma(\bar{\eta}(k))\right) \Gamma(a+\sigma(\varepsilon(k))+\sigma(\bar{l}(k)))}{\Gamma\left(\varepsilon_{k}+\sigma(\eta(k))\right) \Gamma\left(a+\sigma(\varepsilon(k))+s_{k}+\sigma(l(k))\right)} \\
= & \prod_{\nu=0}^{d-1} \frac{a+\sigma(\varepsilon(k))+s_{k}+\sigma(l(k))+\nu}{\varepsilon_{k}+\sigma(\bar{\eta}(k))+\nu} \\
& \geq \exp \left\{\sum_{\nu=0}^{d-1} \frac{a+\sigma(\varepsilon(k))+s_{k}+\sigma(l(k))-\sigma(\bar{\eta}(k))-\varepsilon_{k}}{a+\sigma(\varepsilon(k))+s_{k}+\sigma(l(k))+\nu}\right\} \\
& \geq \exp \left\{\sum_{\nu=0}^{d-1} \frac{s_{k}}{2\left(s_{k}+\sigma(l(k))+\nu\right)}\right\}>\exp \frac{d}{4 n-2} \geq 1
\end{aligned}
$$

since $s_{k} \geq 2 \varepsilon_{k}$ and $e^{x} \leq(1-x)^{-1}$ when $1>x \geq 0$.

Applying this inequality to the $J$, we obtain

$$
\begin{aligned}
J= & \sum_{l(k)+\eta(k)=s(k), l(k) \in \bar{E}} C\left(\varepsilon_{k}, \eta(k)\right) C\left(a+\sigma(\varepsilon(k))+s_{k}, l(k)\right) \\
& \times b\left(a+\sigma(\varepsilon(k)), s_{k}\right) \prod_{j \neq k}\left(1+l_{j}\right)^{-\varepsilon_{j}} \\
\geq & 2^{-\sum_{j \neq k}\left|\varepsilon_{j}\right|} \sum_{l(k)+\eta(k)=s(k), l(k) \in \bar{E}} C\left(\varepsilon_{k}, \eta(k)\right) \\
& \times b\left(a+\sigma(\varepsilon(k))+\sigma(l(k)), s_{k}\right) C(a+\sigma(\varepsilon(k)), l(k)) \prod_{j \neq k}\left(1+s_{j}\right)^{-\varepsilon_{j}} \\
\geq & 2^{1-n-\sum_{j \neq k}\left|\varepsilon_{j}\right|} \prod_{j \neq k}\left(1+s_{j}\right)^{-\varepsilon_{j}} b\left(a+\sigma(\varepsilon(k)), s_{k}\right) \\
& \times \sum_{l(k)+\eta(k)=s(k)} C\left(\varepsilon_{k}, \eta(k)\right) C\left(a+\sigma(\varepsilon(k))+s_{k}, l(k)\right) \\
= & 2^{1-n-\sum_{j \neq k}\left|\varepsilon_{j}\right|} \prod_{j \neq k}\left(1+s_{j}\right)^{-\varepsilon_{j}} b\left(a+\sigma(\varepsilon(k)), s_{k}\right) \\
& \times C\left(a+\sigma(\varepsilon)+s_{k}, s(k)\right)
\end{aligned}
$$

by Lemma 4 .

Using Lemma 6, we have

$$
\begin{aligned}
& \frac{b\left(a+\sigma(\varepsilon(k)), s_{k}\right)\left(1+s_{k}\right)^{\varepsilon_{k}}}{b\left(a+\sigma(\varepsilon), s_{k}\right)} \\
& \quad=\frac{\Gamma\left(a+\sigma(\varepsilon(k))+\varepsilon_{k}\right) \Gamma\left(a+\sigma(\varepsilon(k))+s_{k}\right)\left(1+s_{k}\right)^{\varepsilon_{k}}}{\Gamma(a+\sigma(\varepsilon(k))) \Gamma\left(a+\sigma(\varepsilon(k))+\varepsilon_{k}+s_{k}\right)} \\
& \quad \geq C \frac{\Gamma(a+\sigma(\varepsilon))}{\Gamma(a+\sigma(\varepsilon(k)))}\left(\frac{1+s_{k}}{a+\sigma(\varepsilon)+s_{k}}\right)^{\varepsilon_{k}} \\
& \quad \geq C \frac{\Gamma(a+\sigma(\varepsilon))}{\Gamma(a+\sigma(\varepsilon(k)))} \min \left\{1,\left(\frac{1+2 \varepsilon_{k}}{a+\sigma(\varepsilon)+2 \varepsilon_{k}}\right)\right\} \equiv c_{1}
\end{aligned}
$$


since $s_{k}>2 \varepsilon_{k}$, and $c_{1}$ is a constant which only depends on $a$ and $\varepsilon$. Thus

$$
\begin{aligned}
J & \geq 2^{1-n-\sum_{j \neq k}\left|\varepsilon_{k}\right|} c_{1} \prod_{j=1}^{n}\left(1+s_{j}\right)^{-\varepsilon_{j}} C\left(a+\sigma(\varepsilon)+s_{k}, s(k)\right) b\left(a+\sigma(\varepsilon), s_{k}\right) \\
& =2^{1-n-\sum_{j \neq k}\left|\varepsilon_{j}\right|} c_{1} C(a+\sigma(\varepsilon), s) \prod_{j=1}^{n}\left(1+s_{j}\right)^{-\varepsilon_{j}} \\
& =2^{1-n-\sum_{j \neq k}\left|\varepsilon_{j}\right|} c_{1} A_{s, n}^{a, \varepsilon}
\end{aligned}
$$

by (12) and (18).

We have proved the left-hand inequality of (17) when $\varepsilon_{k}>0$.

We now consider the case 2) $\varepsilon_{k}=0$. The proof of (17) is easy. By (20), we know

$$
B_{s, n}^{a, \varepsilon}=b\left(a+\sigma(\varepsilon(k))+\sigma(s(k)), s_{k}\right) B_{s(k), n-1}^{a, \varepsilon(k)} .
$$

By induction hypothesis, there exist two constants $m_{n-1}$ and $M_{n-1}$, which are independent of $s$, such that

$$
m_{n-1} A_{s(k), n-1}^{a, \varepsilon(k)} \leq B_{s(k), n-1}^{a, \varepsilon(k)} \leq M_{n-1} A_{s(k), n-1}^{a, \varepsilon(k)} .
$$

Thus

$$
\begin{aligned}
B_{s, n}^{a, \varepsilon} & \leq M_{n-1} b\left(a+\sigma(\varepsilon(k))+\sigma(s(k)), s_{k}\right) A_{s(k), n-1}^{a, \varepsilon(k)} \\
& =M_{n-1} b\left(a+\sigma(\varepsilon(k))+\sigma(s(k)), s_{k}\right) C(a+\sigma(\varepsilon(k)), s(k)) \prod_{j \neq k}\left(1+s_{j}\right)^{-\varepsilon_{j}} \\
& =M_{n-1} C(a+\sigma(\varepsilon), s) \prod_{j=1}^{n}\left(1+s_{j}\right)^{-\varepsilon_{j}}=M_{n-1} A_{s, n}^{a, \varepsilon}
\end{aligned}
$$

since $\varepsilon_{k}=0$.

Similarly, we can prove $B_{s, n}^{a, \varepsilon} \geq m_{n-1} A_{s, n}^{a, \varepsilon}$.

\section{Proof of the Main Lemma, Part 2}

In this section we prove the Main Lemma in the final case 3) $\varepsilon_{k}<0$.

We take $a_{k}=-\varepsilon_{k}$ at (22). By Lemma 10, we know $B_{l(k), n-1}^{a-a_{k}, \varepsilon(k)} \geq 0$. All the factors in each term of the right-hand side of the equality (22) are non-negative.

The induction process is as follows: to prove (17) is true if we assume

$$
m_{n-1}^{\prime}(j) A_{l(j), n-1}^{a-a_{k}, \varepsilon(j)} \leq B_{l(j), n-1}^{a-a_{k}, \varepsilon(j)} \leq M_{n-1}^{\prime}(j) A_{l(j), n-1}^{a-a_{k}, \varepsilon(j)}
$$

is true for $j=1,2, \ldots, n$, where $m_{n-1}^{\prime}(j)$ and $M_{n-1}^{\prime}(j)$ are two positive constants, which are independent of $s(j)$, and only depend on $a$ and $\varepsilon(j)$, and

$$
A_{l(j), n-1}^{a-a_{j}, \varepsilon(j)}=C\left(a-a_{j}+\sigma(\varepsilon(j)), l(j)\right) \prod_{j \neq k}\left(1+l_{j}\right)^{-\varepsilon_{j}} .
$$

Let

$$
m_{n-1}^{\prime}=\min _{1 \leq j \leq n} m_{m-1}^{\prime}(j), \quad M_{n-1}^{\prime}=\max _{1 \leq j \leq n} M_{n-1}^{\prime}(j),
$$

then

$$
m_{n-1}^{\prime} A_{l(j), n-1}^{a-a_{j}, \varepsilon(j)} \leq B_{l(j), n-1}^{a-a_{k}, \varepsilon(j)} \leq M_{n-1}^{\prime} A_{l(j), n-1}^{a-a_{j}, \varepsilon(j)} .
$$


In particular, if we take $j=k$, then

$$
m_{n-1}^{\prime} A_{l(k), n-1}^{a-a_{k}, \varepsilon(k)} \leq B_{l(k), n-1}^{a-a_{k}, \varepsilon(k)} \leq M_{n-1}^{\prime} A_{l(k), n-1}^{a-a, \varepsilon(k)} .
$$

Let

$$
P_{s}^{a, \varepsilon}=\sum_{l+\xi=s} C\left(\xi_{k}, \xi(k)\right) b\left(a_{k}, \xi_{k}\right) b\left(a-a_{k}+\sigma(\varepsilon(k))+\sigma(l(k)), l_{k}\right) A_{l(k), n-1}^{a-a_{k}, \varepsilon(k)},
$$

then

$$
m_{n-1}^{\prime} P_{s}^{a, \varepsilon} \leq B_{s, n}^{a, \varepsilon} \leq M_{n-1}^{\prime} P_{s}^{a, \varepsilon}
$$

hold by (22).

We estimate $P_{s}^{a, \varepsilon}$.

Partition the sum $P_{s}^{a, \varepsilon}$ into two parts,

$$
\begin{aligned}
P_{s}^{a, \varepsilon}= & \sum_{l+\xi=s}=\sum_{l_{k}+\xi_{k}=s_{k}, l_{k} \geq \frac{3}{4} s_{k}} \sum_{l(k)+\xi(k)=s(k)}=\sum_{l_{k}+\xi_{k}=s_{k}, l_{k}<\frac{3}{4} s_{k}} \sum_{l(k)+\xi(k)=s(k)}=P_{1}+P_{2} .
\end{aligned}
$$

Let

$$
Q_{l, \xi}^{a, \varepsilon}=C\left(\xi_{k}, \xi(k)\right) b\left(a-a_{k}+\sigma(\varepsilon(k))+\sigma(l(k)), l_{k}\right) A_{l(k), n-1}^{a-a_{k}, \varepsilon(k)},
$$

then

$$
\begin{aligned}
Q_{l, \xi}^{a, \varepsilon}= & C\left(\xi_{k}, \xi(k)\right) b\left(a-a_{k}+\sigma(\varepsilon(k))+\sigma(l(k)), l_{k}\right) C\left(a-a_{k}+\sigma(\varepsilon(k)), l(k)\right) \\
& \times \prod_{j \neq k}\left(1+l_{j}\right)^{-\varepsilon_{j}}=C\left(\xi_{k}, \xi(k)\right) C\left(a-a_{k}+\sigma(\varepsilon(k)), l\right) \prod_{j \neq k}\left(1+l_{j}\right)^{-\varepsilon_{j}}
\end{aligned}
$$

by (12), and

$$
P_{s}^{a, \varepsilon}=\sum_{l+\xi=s} b\left(a_{k}, \xi_{k}\right) Q_{l, \xi}^{a, \varepsilon}=P_{1}+P_{2} .
$$

Let

$$
E_{0}=\left\{l=\left(l_{1}, \ldots, l_{n}\right) \in \mathbf{Z}_{+}^{n}, 0 \leq l_{j} \leq s_{j}, j=1, \ldots, n\right\},
$$

and define the mapping $\phi_{0}: E_{0} \rightarrow E_{0}$, to $\phi_{0}(l)=\bar{l}=\left(\bar{l}_{1}, \ldots, \bar{l}_{n}\right)$ where $\bar{l}_{j}=l_{j}$, if $\frac{1}{2} s_{j} \leq l_{j} \leq s_{j} ; \bar{l}_{j}=s_{j}-l_{j}$, if $0 \leq l_{j}<\frac{1}{2} s_{j}, j=1, \ldots, n$; and define $\bar{\xi}$ as $\bar{\xi}+l=\xi+l=s, \bar{E}_{0}=\phi_{0}\left(E_{0}\right)$.

Consider

$$
G=\frac{Q_{\bar{l}, \bar{\xi}}^{a, \varepsilon}}{Q_{l, \xi}^{a, \varepsilon}}=\frac{C\left(\bar{\xi}_{k}, \bar{\xi}(k)\right) C\left(a-a_{k}+\sigma(\varepsilon(k)), \bar{l}\right)}{C\left(\xi_{k}, \xi(k)\right) C\left(a-a_{k}+\sigma(\varepsilon(k)), l\right)} \prod_{j \neq k}\left(\frac{1+l_{j}}{1+\bar{l}_{j}}\right)^{\varepsilon_{j}} .
$$

When $l_{k} \geq \frac{3}{4} s_{k}$, we have $\bar{l}_{k}=l_{k}, \bar{\xi}_{k}=\xi_{k}$, and then

$$
\begin{aligned}
G & =\frac{\Gamma\left(\sigma(\bar{\xi}) \Gamma\left(a-a_{k}+\sigma(\varepsilon(k))+\sigma(\bar{l})\right)\right.}{\Gamma(\sigma(\xi)) \Gamma\left(a-a_{k}+\sigma(\varepsilon(k))+\sigma(l)\right)} \prod_{j \neq k}\left(\frac{1+l_{j}}{1+\bar{l}_{j}}\right)^{\varepsilon_{j}} \\
& =\prod_{\nu=0}^{d-1} \frac{a-a_{k}+\sigma(\varepsilon(k))+\sigma(l)+\nu}{\sigma(\bar{\xi})+\nu} \prod_{j \neq k}\left(\frac{1+l_{j}}{1+l_{j}+d_{j}}\right)^{\varepsilon_{j}}
\end{aligned}
$$

where $d=\sigma(\bar{l}(k))-\sigma(l(k)), d_{j}=\bar{l}_{j}-l_{j}, j \neq k$. 
By the definition of $\phi_{0}$, we have $\sigma(l(k)) \geq \sigma(\bar{\xi}(k))$, and $l_{k}-\bar{\xi}_{k} \geq \frac{1}{2} s_{k}$ since $l_{k} \geq \frac{3}{4} s_{k}$. By the hypothesis of the Main Lemma and $a_{k}+\varepsilon_{k}=0$, we have $a-a_{k}+\sigma(\varepsilon(k))>0$. The first product in the right-hand side of (29) is equal to

$$
\prod_{\nu=0}^{d-1}\left(1+\frac{a-a_{k}+\sigma(\varepsilon(k))+\sigma(l)-\sigma(\bar{\xi})}{\sigma(\bar{\xi})+\nu}\right) \geq \prod_{\nu=0}^{d-1}\left(1+\frac{\frac{1}{2} s_{k}}{\sigma(\bar{\xi})+\nu}\right)
$$

since $a-a_{k}+\sigma(\varepsilon(k))>0$, and

$$
\sigma(l)-\sigma(\bar{\xi})=l_{k}-\bar{\xi}_{k}+\sigma(l(k))-\sigma(\bar{\xi}(k))>l_{k}-\bar{\xi}_{k} \geq \frac{1}{2} s_{k} .
$$

Moreover,

$$
\sigma(\bar{\xi})+\nu \leq n s_{k}+(n-1) s_{k} \leq(2 n-1) s_{k}
$$

and

$$
\left(\frac{1+l_{j}}{1+l_{j}+d_{j}}\right)^{\varepsilon_{j}} \geq\left(1+d_{j}\right)^{-\left|\varepsilon_{j}\right|} \geq(1+d)^{-\left|\varepsilon_{j}\right|}
$$

hence

$$
\begin{aligned}
G & \geq \prod_{\nu=0}^{d-1}\left(\frac{4 n-1}{4 n-2}\right) \prod_{j \neq k}\left(\frac{1}{1+d}\right)^{\left|\varepsilon_{j}\right|} \\
& =\left(\frac{4 n-1}{4 n-2}\right)^{d}\left(\frac{1}{1+d}\right)^{\sum_{j \neq k}\left|\varepsilon_{j}\right|} \equiv f .
\end{aligned}
$$

Then function $f$ take its minimum value at $d=\left(\log \frac{4 n-1}{4 n-2}\right)^{-1} \sum_{j \neq k}\left|\varepsilon_{j}\right|-1$, thus

$$
\begin{aligned}
G \geq & \left(\frac{4 n-1}{4 n-2}\right)^{\sum_{j \neq k}\left|\varepsilon_{j}\right|\left(\log \left(\frac{4 n-1}{4 n-2}\right)\right)^{-1}-1} \\
& \times\left(\log \left(\frac{4 n-1}{4 n-2}\right)\right)^{\sum_{j \neq k}\left|\varepsilon_{j}\right|}\left(\sum_{j \neq k}\left|\varepsilon_{j}\right|\right)^{-\sum_{j \neq k}\left|\varepsilon_{j}\right|} \equiv a_{0}^{\prime}
\end{aligned}
$$

if $\sum_{j \neq k}\left|\varepsilon_{j}\right| \neq 0$; and $G \geq 1$, if $\sum_{j \neq k}\left|\varepsilon_{j}\right|=0$. Let $a_{1}^{\prime}=\min \left(1, a_{0}^{\prime}\right)$, then $G \geq a_{1}^{\prime}$. Obviously, $a_{1}^{\prime}$ is a constant which only depends on $a$ and $\varepsilon$.

Using the inequality $G \geq a_{1}^{\prime}$ and (29), we have

$$
\begin{aligned}
P_{1} & =\sum_{l_{k}+\xi_{k}=s_{k}, l_{k} \geq \frac{3}{4} s_{k}} \sum_{l(k)+\xi(k)=s(k)} b\left(a_{k}, \xi_{k}\right) Q_{l, \xi}^{a, \varepsilon} \\
& \leq \frac{2^{n-1}}{a_{1}^{\prime}} \sum_{l_{k}+\xi_{k}=s_{k}, l_{k} \geq \frac{3}{4} s_{k}} \sum_{l(k)+\xi(k)=s(k), l \in \bar{E}_{0}} b\left(a_{k}, \xi_{k}\right) Q_{l, \xi}^{a, \varepsilon} .
\end{aligned}
$$

By the definition of $Q_{l, \xi}^{a, \varepsilon}$, and we observe (28) holds true if $l \in \bar{E}_{0}$. Then

$$
2^{-\sum_{j \neq k}\left|\varepsilon_{j}\right|} Q_{0} \leq Q_{l, \xi}^{a, \varepsilon} \leq 2^{\sum_{j \neq k}\left|\varepsilon_{j}\right|} Q_{0}
$$

where

$$
Q_{0}=C\left(\xi_{k}, \xi(k)\right) C\left(a-a_{k}+\sigma(\varepsilon(k)), l\right) \prod_{j \neq k}\left(1+s_{j}\right)^{-\varepsilon_{j}} .
$$


The right-hand inequality implies

$$
\begin{aligned}
P_{1} \leq & C_{2} \sum_{l_{k}+\xi_{k}=s_{k}, l_{k} \geq \frac{3}{4} s_{s_{k}}} \sum_{l(k)+\xi(k)=s(k), l \in \bar{E}_{0}} b\left(a_{k}, \xi_{k}\right) C\left(\xi_{k}, \xi(k)\right) \\
& \times C\left(a-a_{k}+\sigma(\varepsilon(k)), l\right) \prod_{j \neq k}\left(1+s_{j}\right)^{-\varepsilon_{j}} \\
\leq & C_{2} \prod_{j \neq k}\left(1+s_{j}\right)^{-\varepsilon_{j}} \sum_{l+\xi=s} b\left(a_{k}, \xi_{k}\right) C\left(\xi_{k}, \xi(k)\right) C\left(a-a_{k}+\sigma(\varepsilon(k)), l\right) \\
= & C_{2} \prod_{j \neq k}\left(1+s_{j}\right)^{-\varepsilon_{j}} \sum_{l_{k}+\xi_{k}=s_{k}} b\left(a_{k}, \xi_{k}\right) b\left(a-a_{k}+\sigma(\varepsilon(k)), l_{k}\right) \\
& \times \sum_{l(k)+\xi(k)=s(k)} C_{\left(\xi_{k}, \xi(k)\right) C\left(a-a_{k}+\sigma(\varepsilon(k))+l_{k}, l(k)\right)} C_{2} \prod_{j \neq k}\left(1+s_{j}\right)^{-\varepsilon_{j}} \sum_{l_{k}+\xi_{k}=s_{k}} b\left(a_{k}, \xi_{k}\right) b\left(a-a_{k}+\sigma(\varepsilon(k)), l_{k}\right) \\
& \times C_{\left(a+\sigma(\varepsilon)+s_{k}, s(k)\right)} \\
= & C_{2} \prod_{j \neq k}\left(1+s_{j}\right)^{-\varepsilon_{j}} b\left(a+\sigma(\varepsilon(k)), s_{k}\right) C\left(a+\sigma(\varepsilon)+s_{k}, s(k)\right)
\end{aligned}
$$

where $C_{2}=2^{n-1+\sum_{j \neq k}\left|\varepsilon_{j}\right|} / a_{1}^{\prime}$.

Using Lemma 6 , and a similar process as we used in section 4, we may prove

$$
\frac{b\left(a+\sigma(\varepsilon(k)), s_{k}\right)\left(1+s_{k}\right)^{\varepsilon_{k}}}{b\left(a+\sigma(\varepsilon), s_{k}\right)} \leq C_{3}
$$

where $C_{3}$ is a constant, which only depends on $a$ and $\varepsilon$. So we have

$$
\begin{aligned}
P_{1} & \leq C_{2} C_{3} \prod_{j=1}^{n}\left(1+s_{j}\right)^{-\varepsilon_{j}} b\left(a+\sigma(\varepsilon), s_{k}\right) C\left(a+\sigma(\varepsilon)+s_{k}, s(k)\right) \\
& =C_{2} C_{3} A_{s, n}^{a, \varepsilon} .
\end{aligned}
$$

Now we estimate the upper bound of $P_{2}$.

$P_{2}$ is defined as

$$
\begin{gathered}
P_{2}=\sum_{l_{k}+\xi_{k}=s_{k}, l_{k}<\frac{3}{4} s_{k}} \sum_{l(k)+\xi(k)=s(k)} b\left(a_{k}, \xi_{k}\right) C\left(\xi_{k}, \xi(k)\right) \\
\times C\left(a-a_{k}+\sigma(\varepsilon(k)), l\right) \prod_{j \neq k}\left(1+l_{j}\right)^{-\varepsilon_{j}}
\end{gathered}
$$

It is equal to

$$
\begin{gathered}
\sum_{l_{k}+\xi_{k}=s_{k}, l_{k}<\frac{3}{4} s_{k}} \sum_{l(k)+\xi(k)=s(k)} \frac{C\left(\xi_{k}, \xi(k)\right)}{c\left(a_{k}+\xi_{k}, \xi(k)\right)} b\left(a-a_{k}+\sigma(\varepsilon(k))+\sigma(l(k)), l_{k}\right) \\
\times b\left(a_{k}+\sigma(\xi(k)), \xi_{k}\right) C\left(a_{k}, \xi(k)\right) C\left(a-a_{k}+\sigma(\varepsilon(k)), l(k)\right) \prod_{j \neq k}\left(1+l_{j}\right)^{-\varepsilon_{j}}
\end{gathered}
$$

since

$$
b\left(a_{k}, \xi_{k}\right) C\left(a_{k}+\xi_{k}, \xi(k)\right)=b\left(a_{k}+\sigma(\xi(k)), \xi_{k}\right) C\left(a_{k}, \xi(k)\right)
$$


by (12). Let $r_{j}=\left\{\varepsilon_{j}\right\}, j=1, \ldots, n$, where $\{x\}$ means the least integer $X$, such that $X \geq x$, and $r=\left(r_{1}, \ldots, r_{n}\right), \bar{\varepsilon}_{j}=\varepsilon_{j}-\left\{\varepsilon_{j}\right\}$, then $-1<\bar{\varepsilon}_{j} \leq 0, j \neq k$, and $\bar{\varepsilon}_{k}=\varepsilon_{k}$ since $-1<\varepsilon_{k} \leq 0$.

Using Lemma 6, we have

$$
\begin{aligned}
\frac{\Gamma\left(1+l_{j}+r_{j}\right)\left(1+l_{j}+r_{j}\right)^{\bar{\varepsilon}_{j}}}{\Gamma\left(1+l_{j}\right)\left(1+l_{j}\right)^{\varepsilon_{j}}} & \leq C_{4 j} \frac{\left(1+l_{j}\right)^{r_{j}}\left(1+l_{j}+r_{j}\right)^{\bar{\varepsilon}_{j}}}{\left(1+l_{j}\right)^{\varepsilon_{j}}} \\
=C_{4 j}\left(\frac{1+l_{j}+r_{j}}{1+l_{j}}\right)^{\bar{\varepsilon}_{j}} & \leq C_{4 j},
\end{aligned}
$$

and

$$
\begin{gathered}
\frac{\Gamma\left(1+l_{j}+r_{j}\right)\left(1+l_{j}+r_{j}\right)^{\bar{\varepsilon}_{j}}}{\Gamma\left(1+l_{j}\right)\left(1+l_{j}\right)^{\varepsilon_{j}}} \geq c_{3 j}\left(\frac{1+l_{j}+r_{j}}{1+l_{j}}\right)^{\bar{\varepsilon}_{j}} \\
\geq c_{3 j}\left(1+r_{j}\right)^{\bar{\varepsilon}_{j}}=c_{4 j}
\end{gathered}
$$

where $C_{4 j}, c_{3 j}$ and $c_{4 j}$ are constants, which only depend on $\varepsilon_{j}$. Let

$$
C_{4}=\max _{j \neq k} C_{4 j}, \quad c_{4}=\min _{j \neq k} c_{4 j} .
$$

Thus we have

$$
\begin{gathered}
c_{4}\left(1+l_{j}+r_{j}\right)^{-\bar{\varepsilon}_{j}} \frac{\Gamma\left(1+l_{j}\right)}{\Gamma\left(1+l_{j}+r_{j}\right)} \leq\left(1+l_{j}\right)^{-\varepsilon_{j}} \\
\leq C_{4}\left(1+l_{j}+r_{j}\right)^{-\bar{\varepsilon}_{j}} \frac{\Gamma\left(1+l_{j}\right)}{\Gamma\left(1+l_{j}+r_{j}\right)} .
\end{gathered}
$$

Let $q=l+r$, then

$$
\begin{aligned}
C(a- & \left.a_{k}+\sigma(\varepsilon(k)), l\right) \prod_{j \neq k}\left(1+l_{j}\right)^{-\varepsilon_{j}} \\
& \leq C_{4} \frac{\Gamma(a+\sigma(\varepsilon)+\sigma(l)}{\Gamma(a+\sigma(\varepsilon))} \prod \frac{\left(1+q_{j}\right)^{-\bar{\varepsilon}_{j}}}{\Gamma\left(1+q_{j}\right)} \\
& =C_{5} \frac{\Gamma(a+\sigma(\varepsilon)+\sigma(q)-\sigma(r))}{\Gamma(a+\sigma(\varepsilon)-\sigma(r)) \prod \Gamma\left(1+q_{j}\right)} \prod_{j \neq k}\left(1+q_{j}\right)^{-\bar{\varepsilon}_{j}} \\
& =C_{5} C(a+\sigma(\varepsilon)-\sigma(r), q) \prod_{j \neq k}\left(1+q_{j}\right)^{-\bar{\varepsilon}_{j}}
\end{aligned}
$$

and

$$
C(a+\sigma(\varepsilon), l) \prod_{j \neq k}\left(1+l_{j}\right)^{-\varepsilon_{j}} \geq c_{5} C(a+\sigma(\varepsilon)-\sigma(r), q) \prod_{j \neq k}\left(1+q_{j}\right)^{-\bar{\varepsilon}_{j}}
$$

where

$$
C_{5}=C_{4} \frac{\Gamma(a+\sigma(\varepsilon)-\sigma(r))}{\Gamma(a+\sigma(\varepsilon))}, \quad c_{5}=c_{4} \frac{\Gamma(a+\sigma(\varepsilon)-\sigma(r))}{\Gamma(a+\sigma(\varepsilon))} .
$$

Define the mapping $\phi^{*}: E \rightarrow E$, by $l(k) \rightarrow \bar{l}(k)=\phi^{*}(l(k))$, where $\bar{l}_{j}=l_{j}$, if $\frac{1}{2}\left(s_{j}-r_{j}\right)<l_{j} \leq s_{j}$; and $\bar{l}_{j}=s_{j}-l_{j}$, if $0 \leq l_{j} \leq \frac{1}{2}\left(s_{j}-r_{j}\right)$.

Let $E^{*}=\phi^{*}(E)$. Define $\xi, \bar{l}$ and $\bar{\xi}$ by $l+\xi=s, \bar{l}=\phi^{*}(l)$ and $\bar{l}+\bar{\xi}=l+\xi=s$. Consider 


$$
R=\frac{C\left(a_{k}, \bar{\xi}\right) C(a+\sigma(\varepsilon), \bar{l})}{C\left(a_{k}, \xi\right) C(a+\sigma(\varepsilon), l)} \prod_{j \neq k}\left(\frac{1+l_{j}}{1+\bar{l}_{j}}\right)^{\varepsilon_{j}} .
$$

Then

$$
R \geq{ }_{C_{5}} \frac{C\left(a_{k}, \bar{\xi}\right) C(a+\sigma(\varepsilon)-\sigma(r), \bar{q})}{C\left(a_{k}, \xi\right) C(a+\sigma(\varepsilon)-\sigma(r), q)} \prod_{j \neq k}\left(\frac{1+\bar{q}_{j}}{1+q_{j}}\right)^{-\bar{\varepsilon}_{j}}
$$

where

$$
\bar{q}+\bar{\xi}=q+\xi=l+r+\xi=s+r=\bar{l}+r+\bar{\xi} .
$$

By the definition of $\phi^{*}$ and $\bar{q}$, we have $q_{j} \leq \bar{q}_{j}$, hence

$$
\prod_{j \neq k}\left(\frac{1+\bar{q}_{j}}{1+q_{j}}\right)^{-\bar{\varepsilon}_{j}} \geq 1
$$

and

$$
\begin{aligned}
R & \geq c_{6} \frac{C\left(a_{k}, \bar{\xi}\right) C(a+\sigma(\varepsilon)-\sigma(r), \bar{q})}{C\left(a_{k}, \xi\right) C(a+\sigma(\varepsilon)-\sigma(r), q)} \\
& =c_{6} \frac{\Gamma\left(\sigma(\bar{\xi})+a_{k}\right) \Gamma(a+\sigma(\varepsilon)-\sigma(r)+\sigma(\bar{q}))}{\Gamma\left(\sigma(\xi)+a_{k}\right) \Gamma(a+\sigma(\varepsilon)-\sigma(r)+\sigma(q))} \\
& \geq c_{6} \frac{\Gamma\left(\sigma\left(\bar{\xi}+a_{k}\right)\right) \Gamma(a+\sigma(\varepsilon)-\sigma(r)+\sigma(\bar{q}))}{\Gamma\left(\sigma(\xi)+a_{k}\right) \Gamma(a+\sigma(\varepsilon)-\sigma(r)+\sigma(q))} \\
& =c_{6} \prod_{\nu=1}^{d-1} \frac{a+\sigma(\varepsilon)-\sigma(r)+\sigma(q)+\nu}{a_{k}+\sigma(\bar{\xi})+\nu} \geq c_{6}
\end{aligned}
$$

since $\sigma(q) \geq \sigma(\bar{\xi})$ and $a+\sigma(\varepsilon)>\sigma(r)+a_{k}$, where $c_{6}=c_{5} / C_{5}$. Thus

$$
\begin{aligned}
P_{2} \leq & c_{6}^{-1} 2^{n-1} b_{1}^{-1} \sum_{l_{k}+\xi_{k}=s_{k}, l_{k}<\frac{3}{4} s_{k}} \sum_{l(k)+\xi(k)=s(k), l(k) \in E^{*}} b\left(a_{k}, \xi_{k}\right) \\
& \times b\left(a+\sigma(\varepsilon)+\sigma(l(k)), l_{k}\right) C\left(\xi_{k}, \xi(k)\right) C(a+\sigma(\varepsilon), l(k)) \prod_{j \neq k}\left(1+l_{j}\right)^{-\varepsilon_{j}}
\end{aligned}
$$

where

$$
0<b_{1} \leq C\left(\xi_{k}, \xi(k)\right)\left(C\left(a_{k}+\xi_{k}, \xi(k)\right)^{-1} \leq 1 .\right.
$$

Since $l(k) \in E^{*}, s_{j} \geq l_{j}>\frac{1}{2}\left(s_{j}-r_{j}\right)$, we can find a constant $p_{j}>0$, which only depends on $\varepsilon_{j}$, such that $\left(1+l_{j}\right)^{-\varepsilon_{j}} \leq p_{j}^{\left|\varepsilon_{j}\right|}\left(1+s_{j}\right)^{-\varepsilon_{j}}$ hold for all but a finite 
number of values of $s_{j}$. Therefore,

$$
\begin{aligned}
P_{2} \leq & C_{6} \prod_{j \neq k}\left(1+s_{j}\right)^{-\varepsilon_{j}} \sum_{l_{k}+\xi_{k}=s_{k}, l_{k}<\frac{3}{4} s_{k}} \sum_{l(k)+\xi(k)=s(k), l(k) \in E^{*}} b\left(a_{k}, \xi_{k}\right) \\
& \times b\left(a+\sigma(\varepsilon)+\sigma(l(k)), l_{k}\right) C\left(\xi_{k}, \xi(k)\right) C(a+\sigma(\varepsilon), l(k)) \\
\leq & C_{6} \prod_{j \neq k}\left(1+s_{j}\right)^{-\varepsilon_{j}} \sum_{l_{k}+\xi_{k}=s_{k}} \sum_{l(k)+\xi(k)=s(k)} b\left(a_{k}, \xi_{k}\right) b(a+\sigma(\varepsilon) \\
& +\sigma\left(l(k), l_{k}\right) C\left(\xi_{k}, \xi(k)\right) C(a+\sigma(\varepsilon), l(k)) \\
= & C_{6} \prod_{j \neq k}\left(1+s_{j}\right)^{-\varepsilon_{j}} \sum_{l_{k}+\xi_{k}=s_{k}} \sum_{l(k)+\xi(k)=s(k)} b\left(a_{k}, \xi_{k}\right) \\
& \times C\left(a+\sigma(\varepsilon)+l_{k}, l(k)\right) b\left(a+\sigma(\varepsilon), l_{k}\right) C\left(\xi_{k}, \xi(k)\right) \\
= & C_{6} \prod_{j \neq k}\left(1+s_{j}\right)^{-\varepsilon_{j}} \sum_{l_{k}+\xi_{k}=s_{k}} b\left(a_{k}, \xi_{k}\right) b\left(a+\sigma(\varepsilon), l_{k}\right) \\
& \times C_{\left(a+\sigma(\varepsilon)+s_{k}, s(k)\right)} \\
= & C_{6} \prod_{j \neq k}\left(1+s_{j}\right)^{-\varepsilon_{j}} b\left(a+\sigma(\varepsilon(k)), s_{k}\right) C\left(a+\sigma(\varepsilon)+s_{k}, s(k)\right) \\
\leq & C_{6} C_{3} \prod_{j=1}^{n}\left(1+s_{j}\right)^{-\varepsilon_{j}} b\left(a+\sigma(\varepsilon), s_{k}\right) C\left(a+\sigma(\varepsilon)+s_{k}, s(k)\right) \\
= & C_{6} C_{3} A_{s, n}^{a, \varepsilon}
\end{aligned}
$$

where $C_{6}=c_{6}^{-1} 2^{n-1} b_{1}^{-1} \prod_{j \neq k} p_{j}^{\left|\varepsilon_{j}\right|}$.

We have proved the right-hand inequality of (17) when $\varepsilon_{k}<0$.

Now we are going to prove the left-hand inequality of (17) when $\varepsilon_{k}<0$. We just need to estimate the lower bound of $P_{s}^{a, \varepsilon}$ when $\varepsilon_{k}<0$.

We already know

$$
\begin{aligned}
P_{s}^{a, \varepsilon}= & \sum_{l+\xi=s} b\left(a_{k}, \xi_{k}\right) Q_{l, \xi}^{a, \varepsilon} \\
= & \sum_{l_{k}+\xi_{k}=s_{k}} \sum_{l(k)+\xi(k)=s(k)} b\left(a_{k}, \xi_{k}\right) C\left(\xi_{k}, \xi(k)\right) \\
& \times C(a+\sigma(\varepsilon), l) \prod_{j \neq k}\left(1+l_{j}\right)^{-\varepsilon_{j}} \\
\geq & \sum_{l_{k}+\xi_{k}=s_{k}} \sum_{l(k)+\xi(k)=s(k), l(k) \in \bar{E}} b\left(a_{k}, \xi_{k}\right) C\left(\xi_{k}, \xi(k)\right) \\
& \times C(a+\sigma(\varepsilon), l) \prod_{j \neq k}\left(1+l_{j}\right)^{-\varepsilon_{j}}
\end{aligned}
$$

where $\bar{E}=\phi(E)$, and $\phi, E$ are defined in section 4 . If we use inequality (28) and the equality

$$
C(a+\sigma(\varepsilon), l)=b\left(a+\sigma(\varepsilon), l_{k}\right) C\left(a+\sigma(\varepsilon)+l_{k}, l(k)\right),
$$


we have

$$
\begin{aligned}
P_{s}^{a, \varepsilon} \geq & 2^{-\sum_{j \neq k}\left|\varepsilon_{j}\right|} \prod_{j \neq k}\left(1+s_{j}\right)^{-\varepsilon_{j}} \sum_{l_{k}+\xi_{k}=s_{k}} b\left(a_{k}, \xi_{k}\right) \\
& \times b\left(a+\sigma(\varepsilon), l_{k}\right) \sum_{l(k)+\xi(k)=s(k), l(k) \in \bar{E}} C\left(\xi_{k}, \xi(k)\right) C\left(a+\sigma(\varepsilon)+l_{k}, l(k)\right) .
\end{aligned}
$$

Let $\bar{l}(k)=\phi(l(k)), \bar{l}(k)+\bar{\xi}(k)=l(k)+\xi(k)=s(k)$, and consider

$$
\begin{aligned}
H & =\frac{C\left(\xi_{k}, \bar{\xi}(k)\right) C(a+\sigma(\varepsilon), \bar{l})}{C\left(\xi_{k}, \xi(k)\right) C(a+\sigma(\varepsilon), l)} \\
& =\frac{\Gamma(\sigma(\bar{\xi})) \Gamma(a+\sigma(\varepsilon)+\sigma(\bar{l}))}{\Gamma(\sigma(\xi)) \Gamma(a+\sigma(\varepsilon)+\sigma(l))} \\
& =\prod_{\nu=0}^{d-1} \frac{a+\sigma(\varepsilon)+\sigma(l)+\nu}{\sigma(\bar{\xi})+\nu}
\end{aligned}
$$

where $d=\sigma(\bar{l}(k))-\sigma(l(k))$. Then $H \geq 1$ since $a+\sigma(\varepsilon)>0$, and $\sigma(l) \geq \sigma(\bar{\xi})$. Hence

$$
\begin{aligned}
P_{s}^{a, \varepsilon} \geq & c_{7} \prod_{j \neq k}\left(1+s_{j}\right)^{-\varepsilon_{j}} \sum_{l_{k}+\xi_{k}=s_{k}} b\left(a_{k}, \xi_{k}\right) \\
& \times b\left(a+\sigma(\varepsilon), l_{k}\right) \sum_{l(k)+\xi(k)=s(k)} C\left(\xi_{k}, \xi(k)\right) C\left(a+\sigma(\varepsilon)+l_{k}, l(k)\right) \\
= & c_{7} \prod_{j \neq k}\left(1+s_{j}\right)^{-\varepsilon_{j}} \sum_{l_{k}+\xi_{k}=s_{k}} b\left(a_{k}, \xi_{k}\right) \\
& \times b\left(a+\sigma(\varepsilon), l_{k}\right) C\left(a+\sigma(\varepsilon)+s_{k}, s(k)\right) \\
= & c_{7} \prod_{j \neq k}\left(1+s_{j}\right)^{-\varepsilon_{j}} b\left(a+\sigma(\varepsilon(k)), s_{k}\right) C\left(a+\sigma(\varepsilon)+s_{k}, s(k)\right) \\
\geq & c_{7} c_{1} \prod_{j=1}^{n}\left(1+s_{j}\right)^{-\varepsilon_{j}} b\left(a+\sigma(\varepsilon), s_{k}\right) C\left(a+\sigma(\varepsilon)+s_{k}, s(k)\right) \\
= & c_{7} c_{1} A_{s, n}^{a \varepsilon}
\end{aligned}
$$

where $c_{7}=2^{1-n-\sum_{j \neq k}\left|\varepsilon_{j}\right|}$ and $c_{1}$ is defined in section 4 .

We have proved the left-hand inequality of (17) when $\varepsilon_{k}<0$.

\section{Proof of the Theorem, Part 1}

Let $D$ be defined as in (1), and its Bergman kernel function be

$$
K(z, \bar{w})=\sum_{m \in \mathbf{Z}_{+}^{n}} C_{m} z^{m} \bar{w}^{m}
$$

where $m=\left(m_{1}, \ldots, m_{n}\right), z=\left(z_{1}, \ldots, z_{n}\right) \in D, w=\left(w_{1}, \ldots, w_{n}\right) \in D, z^{m}=$ $z_{1}^{m_{1}} \cdots z_{n}^{m_{n}}$ and $w^{m}=w_{1}^{m_{1}} \cdots w_{n}^{m_{n}}$. We know

$$
C_{m}=\frac{1}{\pi^{n}\left(\prod_{j=1}^{n} \alpha_{j}\right)} \frac{\Gamma(\sigma(\alpha m)+\sigma(\alpha)+1)}{\prod_{j=1}^{n} \Gamma\left(\alpha_{j} m_{j}+\alpha_{j}\right)}
$$

by (4). 
Lemma 11. There exist two constants $c_{8}$ and $C_{8}$, which are independent of $m$, and depend only on $\alpha$ and $n$, such that

$$
\begin{gathered}
c_{8} B_{[\alpha m], n}^{n+1, \alpha-1} \prod_{j=1}^{n}\left(\frac{1+\sigma(\alpha)+\sigma([\alpha m])}{1+\left[\alpha_{j} m_{j}\right]}\right)^{\varepsilon_{j}} \leq C_{m} \\
\leq C_{8} B_{[\alpha m], n}^{n+1, \alpha-1} \prod_{j=1}^{n}\left(\frac{1+\sigma(\alpha)+\sigma([\alpha m])}{1+\left[\alpha_{j} m_{j}\right]}\right)^{\varepsilon_{j}}
\end{gathered}
$$

where $[x]$ means the largest integer $X$, such that $X \leq x, \varepsilon_{j}=\alpha_{j} m_{j}-\left[\alpha_{j} m_{j}\right]$, and $\sigma([\alpha m])=\sum_{j=1}^{n}\left[\alpha_{j} m_{j}\right]$, and hence $0 \leq \varepsilon_{j}<1$.

Proof. By Lemma 6, there exist $c_{8}^{\prime}, c_{8}^{\prime \prime}, C_{8}^{\prime}$ and $C_{8}^{\prime \prime}$, which are independent of $m$, and only depend on $\alpha$ and $n$, such that

$$
\begin{aligned}
c_{8}^{\prime} \Gamma(\sigma([\alpha m])+\sigma(\alpha)+1)(\sigma([\alpha m])+\sigma(\alpha)+1)^{\sigma(\varepsilon)} \\
\quad \leq \Gamma(\sigma([\alpha m])+\sigma(\varepsilon)+\sigma(\alpha)+1) \\
\quad \leq C_{8}^{\prime} \Gamma(\sigma([\alpha m])+\sigma(\alpha)+1)(\sigma([\alpha m])+\sigma(\alpha)+1)^{\sigma(\varepsilon)}
\end{aligned}
$$

and

$$
\begin{aligned}
& c_{8}^{\prime \prime} \Gamma\left(\left[\alpha_{j} m_{j}\right]+1\right)\left(\left[\alpha_{j} m_{j}\right]+1\right)^{\varepsilon_{j}+\alpha_{j}-1} \leq \Gamma\left(\left[\alpha_{j} m_{j}\right]+\varepsilon_{j}+\alpha_{j}\right) \\
& \leq C_{8}^{\prime \prime} \Gamma\left(\left[\alpha_{j} m_{j}\right]+1\right)\left(\left[\alpha_{j} m_{j}\right]+1\right)^{\varepsilon_{j}+\alpha_{j}-1} .
\end{aligned}
$$

Thus we have

$$
\begin{gathered}
\frac{c_{8}^{\prime}}{C_{8}^{\prime \prime}} \frac{1}{\pi^{n}\left(\prod_{j+1}^{n} \alpha_{j}\right)} \frac{\Gamma(\sigma([\alpha m])+\sigma(\alpha)+1)(\sigma([\alpha m])+\sigma(\alpha)+1)^{\sigma(\varepsilon)}}{\prod_{j=1}^{n} \Gamma\left(\left[\alpha_{j} m_{j}\right]+1\right)\left(\left[\alpha_{j} m_{j}\right]+1\right)^{\varepsilon_{j}+\alpha_{j}-1}} \leq C_{m} \\
\quad \leq \frac{C_{8}^{\prime}}{c_{8}^{\prime \prime}} \frac{1}{\pi^{n}\left(\prod_{j=1}^{n} \alpha_{j}\right)} \frac{\Gamma(\sigma([\alpha m])+\sigma(\alpha)+1)(\sigma([\alpha m])+\sigma(\alpha)+1)^{\sigma(\varepsilon)}}{\prod_{j=1}^{n} \Gamma\left(\left[\alpha_{j} m_{j}\right]+1\right)\left(\left[\alpha_{j} m_{j}\right]+1\right)^{\varepsilon_{j}+\alpha_{j}-1}} .
\end{gathered}
$$

By the Main Lemma, we know

$$
m_{n} A_{[\alpha m], n}^{n+1, \alpha-1} \leq B_{[\alpha m], n}^{n+1, \alpha-1} \leq M_{n} A_{[\alpha m], n}^{n+1, \alpha-1},
$$

where $\alpha-1=\left(\alpha_{1}-1, \ldots, \alpha_{n}-1\right), m_{n}$ and $M_{n}$ are two constants, which are independent of $m$, and only depend on $\alpha, n$. According to the definition (18),

$$
A_{[\alpha m], n}^{n+1, \alpha-1}=\frac{\Gamma(\sigma([\alpha m])+\sigma(\alpha)+1)}{\Gamma(1+\sigma(\alpha)) \prod_{j=1}^{n} \Gamma\left(1+\left[\alpha_{j} m_{j}\right]\right)\left(1+\left[\alpha_{j} m_{j}\right]\right)^{\alpha_{j}-1}},
$$

we have

$$
\begin{aligned}
& m_{n} \frac{\Gamma(\sigma([\alpha m])+\sigma(\alpha)+1)(\sigma([\alpha m])+\sigma(\alpha)+1)^{\sigma(\varepsilon)}}{\Gamma(1+\sigma(\alpha)) \prod_{j=1}^{n} \Gamma\left(1+\left[\alpha_{j} m_{j}\right]\right)\left(1+\left[\alpha_{j} m_{j}\right]\right)^{\varepsilon_{j}+\alpha_{j}-1}} \\
& \quad \leq B_{[\alpha m], n}^{n+1, \alpha-1} \prod_{j=1}^{n}\left(\frac{1+\sigma(\alpha)+\sigma([\alpha m])}{1+\left[\alpha_{j} m_{j}\right]}\right)^{\varepsilon_{j}} \\
& \quad \leq M_{n} \frac{\Gamma(\sigma([\alpha m])+\sigma(\alpha)+1)(\sigma([\alpha m])+\sigma(\alpha)+1)^{\sigma(\varepsilon)}}{\Gamma(1+\sigma(\alpha)) \prod_{j=1}^{n} \Gamma\left(1+\left[\alpha_{j} m_{j}\right]\right)\left(1+\left[\alpha_{j} m_{j}\right]\right)\left(1+\left[\alpha_{j} m_{j}\right]\right)^{\varepsilon_{j}+\alpha_{j}-1}} .
\end{aligned}
$$

Thus we prove the lemma. 
Lemma 12. If $\delta=\left(\delta_{1}, \ldots, \delta_{n}\right), \delta_{j}=0$ or $1, j=1,2, \ldots, n$, then there exist two positive constants $c_{9}$ and $C_{9}$, which are independent of $m$, and only depend on $\alpha$ and $n$, such that

$$
\begin{gathered}
c_{9} B_{[\alpha m], n}^{n+1, \alpha-1} \prod_{j=1}^{n}\left(\frac{1+\sigma(\alpha)+\sigma([\alpha m])}{1+\left[\alpha_{j} m_{j}\right]}\right)^{\delta_{j}} \leq B_{[\alpha m], n}^{n+1, \alpha-1+\delta} \\
\leq C_{9} B_{[\alpha m], n}^{n+1, \alpha-1} \prod_{j=1}^{n}\left(\frac{1+\sigma(\alpha)+\sigma([\alpha m])}{1+\left[\alpha_{j} m_{j}\right]}\right)^{\delta_{j}}
\end{gathered}
$$

where $\alpha-1+\delta=\left(\alpha_{1}-1+\delta_{1}, \ldots, \alpha_{n}-1+\delta_{n}\right)$.

Proof. By the Main Lemma, there exist two positive constants $c_{9}^{\prime}$ and $C_{9}^{\prime}$, which are independent of $m$ and $\delta$, and only depend on $\alpha$ and $n$, such that

$$
c_{9}^{\prime} A_{[\alpha m], n}^{n+1, \alpha-1+\delta} \leq B_{[\alpha m], n}^{n+1, \alpha-1+\delta} \leq C_{9}^{\prime} A_{[\alpha m], n}^{n+1, \alpha-1+\delta} .
$$

According to the definition (18),

$$
A_{[\alpha m], n}^{n+1, \alpha-1+\delta}=\frac{\Gamma(1+\sigma(\alpha)+\sigma([\alpha m])+\sigma(\delta))}{\Gamma(1+\sigma(\alpha)+\sigma(\delta)) \prod_{j=1}^{n} \Gamma\left(1+\left[\alpha_{j} m_{j}\right]\right)\left(1+\left[\alpha_{j} m_{j}\right]\right)^{\alpha_{j}-1+\delta_{j}}},
$$

where $\sigma(\delta)=\sum_{j=1}^{n} \delta_{j}$. By Lemma 6 , there exist two positive constants $c_{9}^{\prime \prime}$ and $C_{9}^{\prime \prime}$, which are independent of $m$ and $\delta$, and only depend on $\alpha$ and $n$, such that

$$
\begin{aligned}
& c_{9}^{\prime \prime} \frac{\Gamma(\sigma([\alpha m])+\sigma(\alpha)+1)(\sigma([\alpha m])+\sigma(\alpha)+1)^{\sigma(\delta)}}{\Gamma(1+\sigma(\alpha))(1+\sigma(\delta))^{\sigma(\delta)} \prod_{j=1}^{n} \Gamma\left(1+\left[\alpha_{j} m_{j}\right]\right)\left(1+\left[\alpha_{j} m_{j}\right]\right)^{\alpha_{j}-1+\delta_{j}}} \\
& \quad \leq A_{[\alpha m], n}^{n+1, \alpha-1+\delta} \\
& \quad \leq C_{9}^{\prime \prime} \frac{\Gamma(\sigma([\alpha m])+\sigma(\alpha)+1)(\sigma([\alpha m])+\sigma(\alpha)+1)^{\sigma(\delta)}}{\Gamma(1+\sigma(\alpha))(1+\sigma(\alpha))^{\sigma(\delta)} \prod_{j=1}^{n} \Gamma\left(1+\left[\alpha_{j} m_{j}\right]\right)\left(1+\left[\alpha_{j} m_{j}\right]\right)^{\alpha_{j}-1+\delta_{j}}} .
\end{aligned}
$$

That is

$$
\begin{gathered}
c_{9}^{\prime \prime} B_{[\alpha m], n}^{n+1, \alpha-1} \prod_{j=1}^{n}\left(\frac{\sigma([\alpha m])+\sigma(\alpha)+1}{\left[\alpha_{j} m_{j}\right]+1}\right)^{\delta_{j}}(1+\sigma(\alpha))^{-\sigma(\delta)} \leq A_{[\alpha m], n}^{n+1, \alpha-1+\delta} \\
\leq C_{9}^{\prime \prime} B_{[\alpha m], n}^{n+1, \alpha-1} \prod_{j=1}^{n}\left(\frac{\sigma([\alpha m])+\sigma(\alpha)+1}{\left[\alpha_{j} m_{j}\right]+1}\right)^{\delta_{j}}(1+\sigma(\alpha))^{-\sigma(\delta)} .
\end{gathered}
$$

Thus we have proved the lemma.

From (4), we know

$$
K(z, \bar{z})=\sum_{z \in \mathbf{Z}_{+}^{n}} C_{m}|z|^{2 m}
$$

where $C_{m}$ is given by (31), and $|z|^{2 m}=\left|z_{1}\right|^{2 m_{1}} \cdots\left|z_{n}\right|^{2 m_{n}}$. Let $u_{j}=\left|z_{j}\right|^{2 / \alpha_{j}}$, $j=1,2, \ldots, n,\|z\|_{\alpha}=\sum_{j=1}^{n}\left|z_{j}\right|^{2 / \alpha_{j}}$, then $D=\left\{z \in \mathbf{C}^{n} \mid\|z\|_{\alpha}<1\right\}$ and

$$
K(z, \bar{z})=\sum_{m \in \mathbf{Z}_{+}^{n}} C_{m} u^{m \alpha}
$$

where $u^{m \alpha}=u_{1}^{m_{1} \alpha_{1}} \cdots u_{n}^{m_{n} \alpha_{n}}$. 
Let $K_{\alpha}(u)=\sum_{m \in \mathbf{Z}_{+}^{n}} C_{m} u^{m \alpha}$, then

$$
K_{\alpha}(u)=\sum_{m \in \mathbf{Z}_{+}^{n}} C_{m} u_{1}^{\left[\alpha_{1} m_{1}\right]} \cdots u_{n}^{\left[\alpha_{n} m_{n}\right]} u_{1}^{\varepsilon_{1}} \cdots u_{n}^{\varepsilon_{n}}
$$

where $\varepsilon_{j}=\alpha_{j} m_{j}-\left[\alpha_{j} m_{j}\right]$ and hence $1<\varepsilon_{j} \geq 0$. By Lemma 11,

$$
\begin{aligned}
K_{\alpha}(u) & \leq C_{8} \sum_{m \in \mathbf{Z}_{+}^{n}} B_{[\alpha m], n}^{n+1, \alpha-1} \prod_{j=1}^{n}\left(\frac{1+\sigma(\alpha)+\sigma([\alpha m])}{1+\left[\alpha_{j} m_{j}\right]} u_{j}\right)^{\varepsilon_{j}} u_{1}^{\left[\alpha_{1} m_{1}\right]} \cdots u_{n}^{\left[\alpha_{n} m_{n}\right]} \\
& \leq C_{8} \sum_{m \in \mathbf{Z}_{+}^{n}} B_{[\alpha m], n}^{n+1, \alpha-1} \prod_{j=1}^{n}\left(1+\frac{1+\sigma(\alpha)+\sigma([\alpha m])}{1+\left[\alpha_{j} m_{j}\right]} u_{j}\right) u_{1}^{\left[\alpha_{1} m_{1}\right]} \cdots u_{n}^{\left[\alpha_{n} m_{n}\right]}
\end{aligned}
$$

since $1+x>x^{a}$ if $x>0$ and $0 \leq a \leq 1$.

Let

$$
\prod_{j=1}^{n}\left(1+x_{j}\right)=\sum_{\delta \in Q} x^{\delta}=\sum_{\delta \in Q} x_{1}^{\delta_{1}} \cdots x_{n}^{\delta_{n}}
$$

where $\delta=\left(\delta_{1}, \ldots, \delta_{n}\right), \delta_{j}=0$ or $1, j=1,2, \ldots, n$, and $Q$ is a set of lattice points in $\mathbf{R}_{+}^{n}$, which contains all the lattice points whose components are only 0 or 1 , then

$$
\begin{aligned}
K_{\alpha}(u) & \leq C_{8} \sum_{m \in \mathbf{Z}_{+}^{n}} B_{[\alpha m], n}^{n+1, \alpha-1} u_{1}^{\left[\alpha_{1} m_{1}\right]} \cdots u_{n}^{\left[\alpha_{n} m_{n}\right]} \\
& \times \sum_{\delta \in Q} \prod_{j=1}^{n}\left(\frac{1+\sigma(\alpha)+\sigma([\alpha m])}{1+\left[\alpha_{j} m_{j}\right]}\right)^{\delta_{j}} u^{\delta_{j}} .
\end{aligned}
$$

By Lemma 12, we have

$$
K_{\alpha}(u) \leq \frac{C_{8}}{c_{9}} \sum_{\delta \in Q} u^{\delta} \sum_{m \in \mathbf{Z}_{+}^{n}} B_{[\alpha m], n}^{n+1, \alpha-1+\delta} u_{1}^{\left[\alpha_{1} m_{1}\right]} \cdots u_{n}^{\left[\alpha_{n} m_{n}\right]}
$$

where $u^{\delta}=u_{1}^{\delta_{1}} \cdots u_{n}^{\delta_{n}}$.

Let $h=\left(h_{1}, \ldots, h_{n}\right) \in \mathbf{Z}_{+}^{n}$ be given. Then as an equation of $m_{j},\left[\alpha_{j} m_{j}\right]=h_{j}$ at most has $\left\{\frac{1}{\alpha_{j}}\right\}$ solutions, $j=1,2, \ldots, n$, where $\{x\}$ means the least integer $X$ such that $x \leq X$. Thus

$$
K_{\alpha}(u) \leq \frac{C_{8}}{c_{9}} \prod_{j=1}^{n}\left\{\frac{1}{\alpha_{j}}\right\} \sum_{\delta \in Q} u^{\delta} \sum_{h \in \mathbf{Z}_{+}^{n}} B_{h, n}^{n+1, \alpha-1+\delta} u_{1}^{h_{1}} \cdots u_{n}^{h_{n}} .
$$

By the definition of $F,(15)$ and (16), we have

$$
\begin{aligned}
\sum_{h \in \mathbf{Z}_{+}^{n}} B_{h, n}^{n+1, \alpha-1+\delta} u_{1}^{h_{1}} \cdots u_{n}^{h_{n}}=F_{n}^{n+1, \alpha-1+\delta}(u) \\
=(1-\sigma(u))^{-n-1} \prod_{j=1}^{n}\left(1+u_{j}-\sigma(u)\right)^{-\alpha_{j}+1-\delta_{j}} .
\end{aligned}
$$


Since $\frac{u_{j}}{1+u_{j}-\sigma(u)}<1$ and $\delta_{j}=0$ or 1 , we have

$$
\begin{aligned}
K_{\alpha}(u) \leq & C_{10} \sum_{\delta \in Q} u^{\delta} F_{n}^{n+1, \alpha-1+\delta}(u) \\
= & C_{10} \sum_{\delta \in Q}(1-\sigma(u))^{-n-1} \prod_{j=1}^{n}\left(\frac{u_{j}}{1+u_{j}-\sigma(u)}\right)^{\delta_{j}} \\
& \times \prod_{j=1}^{n}\left(1+u_{j}-\sigma(u)\right)^{1-\alpha_{j}} \\
= & C_{10} \sum_{\delta \in Q} F_{n}^{n+1, \alpha-1}(u) \prod_{j=1}^{n}\left(\frac{u_{j}}{1+u_{j}-\sigma(u)}\right)^{\delta_{j}} \\
\leq & 2^{n} C_{10} F_{n}^{n+1, \alpha-1}(u)=2^{n} C_{10} F(z, \bar{z})
\end{aligned}
$$

where

$$
C_{10}=\frac{C_{8}}{c_{9}} \prod_{j=1}^{n}\left\{\frac{1}{\alpha_{j}}\right\} .
$$

We have proved the right-hand inequality of (2).

\section{Proof of Theorem, Part 2}

The proof of the left-hand inequality of (2) is little bit difficult.

For any $\delta=\left(\delta_{1}, \ldots, \delta_{n}\right) \in Q$, we define

$$
\phi_{\delta}(m)=\left(\phi_{\delta, 1}\left(m_{1}\right), \ldots, \phi_{\delta, n}\left(m_{n}\right)\right)
$$

as $\phi_{\delta, j}\left(m_{j}\right)=\left[m_{j} \alpha_{j}\right]$ when $\delta_{j}=1 ;$ and $\phi_{\delta, j}\left(m_{j}\right)=\left\{m_{j} \alpha_{j}\right\}$ when $\delta_{j}=0 ; j=$ $1,2, \ldots, n$; where $Q$ is defined in section 6 .

For any $h \in \mathbf{Z}_{+}^{n}, m \in \mathbf{Z}_{+}^{n}$, and $\delta \in Q$, we define $N_{\delta}(m, h)=1$, if $h=\phi_{\delta}(m)$; and $N_{\delta}(m, h)=0$ if $h \neq \phi_{\delta}(m)$, then we have

(1) for a fixed $m \in \mathbf{Z}_{+}^{n}$,

$$
\sum_{h \in \mathbf{Z}_{+}^{n}} N_{\delta}(m, h)=1
$$

(2) for a fixed $h \in \mathbf{Z}_{+}^{n}$,

$$
0 \leq \sum_{m \in \mathbf{Z}_{+}^{n}} N_{\delta}(m, h) \equiv N_{\delta}(h) \leq \prod_{j=1}^{n}\left\{\frac{1}{\alpha_{j}}\right\}
$$

since each of the equations $\left[\alpha_{j} m_{j}\right]=h_{j}$ and $\left\{\alpha_{j} m_{j}\right\}=h_{j}$ has at most $\left\{1 / \alpha_{j}\right\}$ integer solutions for $m_{j}$.

For any $m \in \mathbf{Z}_{+}^{n}$ and $\delta \in Q$, we may decompose $\alpha m$ as

$$
\begin{aligned}
\alpha m & =\left(\alpha_{1} m_{1}, \ldots, \alpha_{n} m_{n}\right) \\
& =\left(\phi_{\delta, 1}\left(m_{1}\right)+\varepsilon_{1}\left(\delta_{1}, m_{1}\right), \ldots, \phi_{\delta, n}\left(m_{n}\right)+\varepsilon_{n}\left(\delta_{n}, m_{n}\right)\right),
\end{aligned}
$$

where

$$
\varepsilon_{j}\left(\delta_{j}, m_{j}\right)=\alpha_{j} m_{j}-\phi_{\delta, j}\left(m_{j}\right)=\alpha_{j} m_{j}-\left[\alpha_{j} m_{j}\right]
$$

if $\delta_{j}=1$; and

$$
\varepsilon_{j}\left(\delta_{j}, m_{j}\right)=\alpha_{j} m_{j}-\phi_{\delta, j}\left(m_{j}\right)=\alpha_{j} m_{j}-\left\{\alpha_{j} m_{j}\right\}
$$


if $\delta_{j}=0 ; j=1,2, \ldots, n$.

Let $\phi_{\delta, j}\left(m_{j}\right)=h_{j}, \varepsilon_{j}=\varepsilon_{j}\left(\delta_{j}, m_{j}\right)$, then

$$
\alpha_{j} m_{j}=\phi_{\delta, j}\left(m_{j}\right)+\varepsilon_{j}\left(\delta_{j}, m_{j}\right)=h_{j}+\varepsilon_{j} .
$$

We may extend Lemma 11 as

Lemma 13. If $\phi_{\delta}(h)=m$, then there exist two positive constants $c_{11}$ and $C_{11}$, which are independent of $m$ and $\delta$, and only depend on $\alpha$ and $n$, such that

$$
\begin{gathered}
c_{11} B_{h, n}^{n+1, \alpha-1} \prod_{j=1}^{n}\left(\frac{1+\sigma(\alpha)+\sigma(h)}{h_{j}+1}\right)^{\varepsilon_{j}} \leq C_{m} \\
\leq C_{11} B_{h, n}^{n+1, \alpha-1} \prod_{j=1}^{n}\left(\frac{1+\sigma(\alpha)+\sigma(h)}{h_{j}+1}\right)^{\varepsilon_{j}} .
\end{gathered}
$$

Since the proof of Lemma 13 is similar to that of Lemma 11, we omit it.

By (32), we have

$$
K_{\alpha}(u)=\sum_{m \in \mathbf{Z}_{+}^{n}} \sum_{h \in \mathbf{Z}_{+}^{n}} N_{\delta}(m, h) C_{m} u_{1}^{h_{1}} \cdots u_{n}^{h_{n}} u_{1}^{\varepsilon_{1}} \cdots u_{n}^{\varepsilon_{n}} .
$$

Using Lemma 13, we find that

$$
K_{\alpha} \geq c_{11} \sum_{m, h \in \mathbf{Z}_{+}^{n}} N_{\delta}(m, h) B_{h, n}^{n+1, \alpha-1} \prod_{j=1}^{n}\left(\frac{\sigma(h)+\sigma(\alpha)+1}{h_{j}+1}\right)^{\varepsilon_{j}} u^{h}
$$

holds for any $\delta \in Q$. Since the total number of lattice points in $Q$ is $2^{n}$, we have

$$
\begin{aligned}
K_{\alpha}(u) & \geq 2^{-n} c_{11} \sum_{h \in \mathbf{Z}_{+}^{n}} B_{h, n}^{n+1, \alpha-1} u^{h} \sum_{\delta \in Q} \\
\times & \left(\sum_{m \in \mathbf{Z}_{+}^{n}} N_{\delta}(m, h) \prod_{j=1}^{n}\left(\frac{\sigma(h)+\sigma(\alpha)+1}{h_{j}+1} u_{j}\right)^{\varepsilon_{j}\left(\delta_{j}, m_{j}\right)}\right) .
\end{aligned}
$$

We consider the last bracket on the right-hand side of the previous inequality. For a fixed $h \in \mathbf{Z}_{+}^{n}$, and a fixed $\delta \in Q$, as an equation in $m, \phi_{\delta}(m)=h$ may have solutions, that means, $h \in \phi_{\delta}\left(\mathbf{Z}_{+}^{n}\right)$; or may not have a solution, that means, $h$ does not belong to $\phi_{\delta}\left(\mathbf{Z}_{+}^{n}\right)$. We define $\bar{N}_{\delta}(h)$ as the characteristic function of $\phi_{\delta}\left(\mathbf{Z}_{+}^{n}\right)$ when $\delta$ is fixed, that is, $\bar{N}_{\delta}(h)=1$ if $h \in \phi_{\delta}\left(\mathbf{Z}_{+}^{n}\right)$; and $\bar{N}_{\delta}(h)=0$, otherwise, Then

$$
K_{\alpha}(u)>2^{-n} c_{11} \sum_{h \in \mathbf{Z}_{+}^{N}} B_{h, n}^{n+1, \alpha-1} \sum_{\delta \in Q} \bar{N}_{\delta}(h) \prod_{j=1}^{n}\left(\frac{\sigma(h)+\sigma(\alpha)+1}{h_{j}+1} u_{j}\right)^{\varepsilon_{j}\left(\delta_{j}, h_{j}\right)}
$$

where $\varepsilon_{j}\left(\delta_{j}, h_{j}\right)=\varepsilon_{j}\left(\delta_{j}, m_{j}\right)$ if $m_{j}$ is a solution of $h_{j}=\phi_{\delta, j}\left(m_{j}\right), j=1,2, \ldots, n$.

Obviously, $\phi\left(\mathbf{Z}_{+}^{n}\right)$ has the following properties:

(1) If $h \in \phi_{\delta}\left(\mathbf{Z}_{+}^{n}\right)$, then $h(j)=\left(h_{1}, \ldots, h_{j-1}, 0, h_{j+1}, \ldots, h_{n}\right) \in \phi_{\delta}\left(\mathbf{Z}_{+}^{n}\right)$;

(2) If $h \in \phi_{\delta}\left(\mathbf{Z}_{+}^{n}\right)$, then there exists an integer $k_{j}, 1 \leq k_{j} \leq\left\{\alpha_{j}\right\}$, such that $h+k_{j} e_{j} \in \phi_{\delta}\left(\mathbf{Z}_{+}^{n}\right)$.

Fix $h(1) \in \phi_{\delta}\left(\mathbf{Z}_{+}^{n}\right)$, then there exists an integer sequence

$$
n_{1,0}=0<n_{1,1}<n_{1,2}<\cdots<n_{1, k}<\cdots, \quad k=0,1,2, \ldots,
$$

which is independent of the choice of $h(1)$, such that $h(1)+n_{1, k} e_{1} \in \phi_{\delta}\left(\mathbf{Z}_{+}^{n}\right)$, and $1 \leq n_{1, k+1}-n_{1, k} \leq\left\{\alpha_{1}\right\}$. Moreover, if $n_{1, k+1} \geq n_{1, k}+2$ holds, then $h(1)+$ $\left(n_{1, k}+s\right) e_{1}$ does not belong to $\phi_{\delta}\left(\mathbf{Z}_{+}^{n}\right)$, when $1 \leq s \leq n_{1, k+1}-n_{1, k}-1$. 
Fix $\delta \in Q$, and consider a subset $D_{1}(\delta) \subset \mathbf{Z}_{+}^{n}$ as

$$
D_{1}(\delta)=\left\{h(1)+k e_{1} \in \mathbf{Z}_{+}^{n}, \text { for all } h(1) \in \phi_{\delta}\left(\mathbf{Z}_{+}^{n}\right), k=0,1, \ldots\right\},
$$

then $D_{1}(\delta) \supset \phi_{\delta}\left(\mathbf{Z}_{+}^{n}\right)$, and

$$
K_{\alpha}(u)>c_{11} 2^{-n} \sum_{\delta \in Q} \sum_{h \in D_{1}(\delta)} \bar{N}_{\delta}(h) B_{h, n}^{n+1, \alpha-1} u^{h} \prod_{j=1}^{n}\left(\frac{\sigma(h)+\sigma(\alpha)+1}{h_{j}+1} u_{j}\right)^{\varepsilon_{j}\left(\delta_{j}, h_{j}\right)} .
$$

Let $\chi=h(1)+n_{1, k} e_{1} \in \phi_{\delta}\left(\mathbf{Z}_{+}^{n}\right), \tau=h(1)+n_{1, k+1} e_{1} \in \phi_{\delta}\left(\mathbf{Z}_{+}^{n}\right)$ and $l=n_{1, k+1}-n_{1, k}$, $1 \leq s \leq n_{1, k+1}-n_{1, k}-1=l-1$ if $l \geq 2$, then

$$
\begin{aligned}
& \frac{s}{l} B_{\tau, n}^{n+1, \alpha-1} u^{\tau} \prod_{j=1}^{n}\left(\frac{\sigma(\tau)+\sigma(\alpha)+1}{\tau_{j}+1} u_{j}\right)^{\varepsilon_{j}\left(\delta_{j}, \tau_{j}\right)} \\
& \quad+\frac{l-s}{l} B_{\chi, n}^{n+1, \alpha-1} u^{\chi} \prod_{j=1}^{n}\left(\frac{\sigma(\chi)+\sigma(\alpha)+1}{\chi_{j}+1} u_{j}\right)^{\varepsilon_{j}\left(\delta_{j}, \chi_{j}\right)} \\
& \geq\left(B_{\tau, n}^{n+1, \alpha-1}\right)^{s / l}\left(B_{\chi, n}^{n+1, \alpha-1}\right)^{(l-s) / l} u^{(\tau+\varepsilon(\delta, \tau)) s / l+(\chi+\varepsilon(\delta, \chi))^{(l-s) / s}} \\
& \quad \times \prod_{j=1}^{n}\left(\frac{\sigma(\tau)+\sigma(\alpha)+1}{\tau_{j}+1}\right)^{s / l \varepsilon_{j}\left(\delta_{j}, \tau_{j}\right)}\left(\frac{\sigma(\chi)+\sigma(\alpha)+1}{\chi+1}\right)^{(l-s) / s \varepsilon_{j}\left(\delta_{j}, \chi_{j}\right)},
\end{aligned}
$$

since the arithmetic mean is greater or equal to the geometric mean.

According to the Main Lemma, there exist two constants $c_{12}$ and $C_{12}$, which are independent of $\tau$ and $\chi$, and only depend on $n$ and $\alpha$, such that

$$
c_{12} A_{\tau, n}^{n+1, \alpha-1} \leq B_{\tau, n}^{n+1, \alpha-1} \leq C_{12} A_{\tau, n}^{n+1, \alpha-1}
$$

and

$$
c_{12} A_{\chi, n}^{n+1, \alpha-1} \leq B_{\chi, n}^{n+1, \alpha-1} \leq C_{12} A_{\tau, n}^{n+1, \alpha-1}
$$

Let $h_{1}=n_{1, k}+s$, then

$$
\begin{aligned}
A_{\tau, n}^{n+1, \alpha-1} & =\frac{\Gamma\left(\sigma(\alpha)+\sigma(h(1))+n_{1, k+1}+1\right)}{\Gamma(\sigma(\alpha)+1) \prod_{j=2}^{n} \Gamma\left(1+h_{j}\right)\left(1+h_{j}\right)^{\alpha_{j}-1} \Gamma\left(1+n_{1, k+1}\right)\left(1+n_{1, k+1}\right)^{\alpha_{1}-1}} \\
& =\frac{\Gamma(\sigma(\alpha)+\sigma(h)+l-s+1)}{\Gamma(\sigma(\alpha)+1) \prod_{j=2}^{n} \Gamma\left(1+h_{j}\right)\left(1+h_{j}\right)^{\alpha_{j}-1} \Gamma\left(h_{1}+l-s+1\right)\left(h_{1}+l-s+1\right)^{\alpha_{1}-1}} \\
& =A_{h, n}^{n+1, \alpha-1} \frac{(\sigma(\alpha)+\sigma(h)+1) \cdots(\sigma(\alpha)+\sigma(h)+l-s)\left(1+h_{1}\right)^{\alpha_{1}-1}}{\left(h_{1}+1\right) \cdots\left(h_{1}+l-s\right)\left(h_{1}+l-s+1\right)^{\alpha_{1}-1}},
\end{aligned}
$$

and

$$
\begin{aligned}
A_{\chi, n}^{n+1, \alpha-1}= & \frac{\Gamma\left(\sigma(\alpha)+\sigma(h(1))+n_{1, k}+1\right)}{\Gamma(\sigma(\alpha)+1) \prod_{j=2}^{n} \Gamma\left(1+h_{j}\right)\left(1+h_{j}\right)^{\alpha_{j}-1} \Gamma\left(1+n_{1, k}\right)\left(1+n_{1, k}\right)^{\alpha_{1}-1}} \\
= & \frac{\Gamma(\sigma(\alpha)+\sigma(h)+1)}{\Gamma(\sigma(\alpha)+1) \prod_{j=2}^{n} \Gamma\left(1+h_{j}\right)\left(1+h_{j}\right)^{\alpha_{j}-1} \Gamma\left(1+h_{1}\right)\left(1+n_{1, k}\right)^{\alpha_{1}-1}} \\
& \times \frac{\left(1+n_{1, k}\right) \cdots\left(s+n_{1, k}\right)}{\left(\sigma(\alpha)+\sigma(h(1))+n_{1, k}+1\right) \cdots\left(\sigma(\alpha)+\sigma(h(1))+n_{1, k}+s\right)} \\
= & A_{h, n}^{n+1, \alpha-1} \frac{\left(1+n_{1, k}\right) \cdots\left(s+n_{1, k}\right)\left(1+h_{1}\right)^{\alpha_{1}-1}}{\left(\sigma(\alpha)+\sigma(h(1))+n_{1, k}+1\right) \cdots\left(\sigma(\alpha)+\sigma(h(1))+n_{1, k}+s\right)\left(1+n_{1, k}\right)^{\alpha_{1}-1}} .
\end{aligned}
$$


Thus

$$
\begin{aligned}
& \left(A_{\tau, n}^{n+1, \alpha-1}\right)^{s / l}\left(A_{\chi, n}^{n+1, \alpha-1}\right)^{(l-s) / l}=A_{h, n}^{n+1, \alpha-1}\left(1+h_{1}\right)^{\alpha_{1}-1} \\
& \quad \times\left(\frac{\sigma(\alpha)+\sigma(h)+1) \cdots(\sigma(\alpha)+\sigma(h)+l-s)}{\left(h_{1}+1\right) \cdots\left(h_{1}+l-s\right)\left(h_{1}+l-s+1\right)^{\alpha_{1}-1}}\right)^{s / l} \\
& \quad \times\left(\frac{\left(1+n_{1, k}\right) \cdots\left(s+n_{1, k}\right)}{\left(\sigma(\alpha)+\sigma(h(1))+n_{1, k}+1\right) \cdots\left(\sigma(\alpha)+\sigma(h(1))+n_{1, k}+s\right)\left(1+n_{1, k}\right)^{\alpha_{1}-1}}\right)^{(l-s) / l} \\
& =A_{h, n}^{n+1, \alpha-1} I_{1} I_{2} I_{3},
\end{aligned}
$$

where

$$
\begin{aligned}
I_{1} & =\frac{\left(1+h_{1}\right)^{\alpha_{1}-1}}{\left(\left(h_{1}+l-s+1\right)^{s / l}\left(h_{1}-s+1\right)^{(l-s) / l}\right)^{\alpha_{1}-1}}, \\
I_{2} & =\frac{((\sigma(\alpha)+\sigma(h)+1) \cdots(\sigma(\alpha)+\sigma(h)+l-s))^{s / l}}{((\sigma(\alpha)+\sigma(h)-s+1) \cdots(\sigma(\alpha)+\sigma(h)))^{(l-s) / l}}
\end{aligned}
$$

and

$$
I_{3}=\frac{\left(h_{1}\left(h_{1}-1\right) \cdots\left(h_{1}-s+1\right)\right)^{(l-s) / l}}{\left(\left(h_{1}+1\right) \cdots\left(h_{1}+l-s\right)\right)^{s / l}} .
$$

Obviously, $I_{1}, I_{2}$ and $I_{3}$ are bounded above and below since $l \leq\left\{\alpha_{1}\right\}$ and $s \leq$ $l-1 \leq\left\{\alpha_{1}\right\}-1$. Thus there exist two positive constants $c_{14}$ and $C_{14}$, which are independent of $\tau, \chi$ and $h$, and only depend on $\alpha$ and $n$, such that

$$
c_{14} B_{h, n}^{n+1, \alpha-1} \leq\left(B_{\tau, n}^{n+1, \alpha-1}\right)^{s / l}\left(B_{\chi, n}^{n+1, \alpha-1}\right)^{(l-s) / l} \leq C_{14} B_{h, n}^{n+1, \alpha-1}
$$

where $1 \leq s \leq n_{1, k+1}-n_{1, k}-1$.

Moreover, we have

$$
\begin{aligned}
\tau \frac{s}{l}+\chi \frac{l-s}{l} & =\left(h(1)+n_{1, k+1} e_{1}\right) \frac{s}{l}+\left(h(1)+n_{1, k} e_{1}\right) \frac{l-s}{l} \\
& =h(1)+\left(n_{1, k}+s\right) e_{1}=h,
\end{aligned}
$$

and

$$
\frac{\sigma(\tau)+\sigma(\alpha)+1}{\tau_{j}+1}=\frac{\sigma(h)+\sigma(\alpha)+1+l-s}{h_{j}+1}
$$

if $j \neq 1$, then there exist two positive constants $c_{15}^{\prime}$ and $C_{15}^{\prime}$, which are independent of $h$, and only depend on $\alpha$ and $n$, such that

$$
c_{15}^{\prime} \frac{\sigma(h)+\sigma(\alpha)+1}{h_{j}+1} \leq \frac{\sigma(\tau)+\sigma(\alpha)+1}{\tau_{j}+1} \leq C_{15}^{\prime} \frac{\sigma(h)+\sigma(\alpha)+1}{h_{j}+1}
$$

when $j \neq 1$, since $1 \leq l-s \leq\left\{\alpha_{1}\right\}-1$. When $j=1$, we have

$$
\frac{\sigma(\tau)+\sigma(\alpha)+1}{\tau_{1}+1}=\frac{n_{1, k+1}+\sigma(h(1))+\sigma(\alpha)+1}{n_{1, k+1}+1}=\frac{\sigma(h)+\sigma(\alpha)+1+l-s}{h_{1}+1+l-s},
$$

then there exist two positive constants $c_{15}^{\prime \prime}$ and $C_{15}^{\prime \prime}$, which are independent of $h$ and $\tau$, and only depend on $\alpha$ and $n$, such that

$$
c_{15}^{\prime \prime} \frac{\sigma(h)+\sigma(\alpha)+1}{h_{1}+1} \leq \frac{\sigma(\tau)+\sigma(\alpha)+1}{\tau_{1}+1} \leq C_{15}^{\prime \prime} \frac{\sigma(h)+\sigma(\alpha)+1}{h_{1}+1} .
$$

Thus

$$
c_{15} \frac{\sigma(h)+\sigma(\alpha)+1}{h_{j}+1} \leq \frac{\sigma(\tau)+\sigma(\alpha)+1}{\tau_{j}+1} \leq C_{15} \frac{\sigma(h)+\sigma(\alpha)+1}{h_{j}+1}
$$


hold for $j=1,2, \ldots, n$, where $c_{15}=\min \left(c_{15}^{\prime}, c_{15}^{\prime \prime}\right)$, and $C_{15}=\max \left(C_{15}^{\prime}, C_{15}^{\prime \prime}\right)$. Similarly, the inequalities

$$
c_{16} \frac{\sigma(h)+\sigma(\alpha)+1}{h_{j}+1} \leq \frac{\sigma(\chi)+\sigma(\alpha)+1}{\chi_{j}+1} \leq C_{16} \frac{\sigma(h)+\sigma(\alpha)+1}{h_{j}+1}
$$

holds for $j=1,2, \ldots, n$, where $c_{16}$ and $C_{16}$ are two positive constants, which are independent of $h$, and only depend on $\alpha$ and $n$.

Combining all these results, we get the right-hand side of (33) is not less than

$$
\begin{gathered}
c_{17} B_{h, n}^{n+1, \alpha-1} u^{h} \prod_{j=2}^{n}\left(\frac{\sigma(h)+\sigma(\alpha)+1}{h_{j}+1} u_{j}\right)^{\varepsilon_{j}\left(\delta_{j}, h_{j}\right)} \\
\times\left(\frac{\sigma(h)+\sigma(\alpha)+1}{h_{1}+1} u_{1}\right)^{\varepsilon_{1}^{\prime}\left(\delta_{1}, h_{1}\right)}
\end{gathered}
$$

where $c_{17}=c_{14} c_{15}^{s / l} c_{16}^{(l-s) / l}$, and $\varepsilon_{1}^{\prime}\left(\delta_{1}, h_{1}\right)=\frac{s}{l} \varepsilon_{1}\left(\delta_{1}, \tau_{1}\right)+\frac{l-s}{l} \varepsilon_{1}\left(\delta_{1}, \chi_{1}\right)$.

Let

$$
T(y)=B_{y, n}^{n+1, \alpha-1} u^{y} \prod_{j=1}^{n}\left(\frac{\sigma(y)+\sigma(\alpha)+1}{y_{j}+1} u_{j}\right)^{\varepsilon_{j}\left(\delta_{j}, y_{i}\right)}
$$

where $y=\tau, \chi$ or $h$. Then

$$
\begin{aligned}
T(\tau)+ & T(\chi)=\frac{2}{l-1} \sum_{s=1}^{l-1} \frac{1}{l}(s T(\tau)+(l-s) T(\chi)) \\
= & \frac{1}{2}(T(\tau)+T(\chi))+\frac{1}{4}(T(\tau)+T(\chi))+\frac{1}{2(l-1)} \sum_{s=1}^{l-1} \frac{1}{l}(s T(\tau)+(l-s) T(\chi)) \\
\geq & \frac{1}{2}(T(\tau)+T(\chi))+\frac{1}{4}(T(\tau)+T(\chi)) \\
& +\frac{c_{17}}{2(l-1)} \sum_{s=1}^{l-1} T(h)\left(\frac{\sigma(h)+\sigma(\alpha)+1}{h_{1}+1} u_{1}\right)^{\varepsilon_{1}^{\prime}\left(\delta_{1}, h_{1}\right)-\varepsilon_{1}\left(\delta_{1}, h_{1}\right)} \\
\geq & \frac{1}{2}(T(\tau)+T(\chi))+c_{18} \sum_{s=0}^{l} T(h)\left(\frac{\sigma(h)+\sigma(\alpha)+1}{h_{1}+1} u_{1}\right)^{\varepsilon_{1}^{\prime}\left(\delta_{1}, h_{1}\right)-\varepsilon_{1}\left(\delta_{1}, h_{1}\right)}
\end{aligned}
$$

where $c_{18}=\min \left(\frac{1}{4}, \frac{c_{17}}{2\left(\left\{\alpha_{1}\right\}-1\right)}\right)$. Thus, we have

$$
\begin{aligned}
K_{\alpha}(u) & >2^{-n} c_{11} c_{18} \sum_{\delta \in Q} \sum_{h \in \mathbf{Z}_{+}^{n}} \bar{N}_{\delta}(1, h) B_{h, n}^{n+1, \alpha-1} u^{h} \\
& \times \prod_{j=2}^{n}\left(\frac{\sigma(h)+\sigma(\alpha)+1}{h_{j}+1} u_{j}\right)^{\varepsilon_{j},\left(\delta_{j}, h_{j}\right)}\left(\frac{\sigma(h)+\sigma(\alpha)+1}{h_{1}+1} u_{1}\right)^{\varepsilon_{1}^{\prime}\left(\delta_{1}, h_{1}\right)}
\end{aligned}
$$

where $\bar{N}_{\delta}(1, h)$ is the characteristic function of $D_{1}(\delta)$, that is, $\bar{N}_{\delta}(1, h)=1$, if $h \in D_{1} ; \bar{N}_{\delta}(1, h)=0$, if $h$ does not belong to $D_{1}$.

If $\delta_{1}=1$, then there exist two positive integers $m_{1}$ and $m_{1}^{\prime}$, such that $\left[\alpha_{1} m_{1}^{\prime}\right]=$ $n_{1, k+1}$, and $\left[\alpha_{1} m_{1}\right]=n_{1, k}$. Hence

$$
\varepsilon_{1}\left(1, \tau_{1}\right)=\alpha_{1} m_{1}^{\prime}-\left[\alpha_{1} m_{1}^{\prime}\right]=\alpha_{1} m_{1}^{\prime}-n_{1, k+1} \geq 0,
$$


and

$$
\varepsilon_{1}\left(1, \chi_{1}\right)=\alpha_{1} m_{1}-\left[\alpha_{1} m_{1}\right]=\alpha_{1} m_{1}-n_{1, k} \geq 0
$$

Since by the definition of $\varepsilon_{1}^{\prime}$,

$$
\varepsilon_{1}^{\prime}\left(\delta_{1}, h_{1}\right)=\frac{s}{l} \varepsilon_{1}\left(\delta_{1}, \tau_{1}\right)+\frac{l-s}{l} \varepsilon_{1}\left(\delta_{1}, \chi_{1}\right),
$$

we have $\varepsilon_{1}^{\prime}\left(1, h_{1}\right) \geq 0$. Similarly, we can prove that $\varepsilon_{1}^{\prime}\left(0, h_{1}\right) \leq 0$.

Fix $h(2) \in D_{1}(\delta)$, then there exists a sequence of integers

$$
n_{2,0}=0<n_{2,1}<\cdots<n_{2, k}<\cdots,
$$

which is independent of the choice of $h(2) \in D_{1}(\delta)$, such that $h(2)+n_{2, k} e_{2} \in D_{1}(\delta)$, and $1 \leq n_{2, k+1}-n_{2, k} \leq\left\{\alpha_{2}\right\}$ and $h(2)+\left(n_{2, k}+s\right)$ does not belong to $D_{1}(\delta)$ when $1 \leq s \leq n_{2, k+1}-n_{2, k}-1$ and $n_{2, k+1} \geq n_{2, k}+2$.

Define $D_{2}=D_{2}(\delta)$ by

$$
D_{2}(\delta)=\left\{h(2)+k e_{2} \in \mathbf{Z}_{+}^{n}, \text { for all } h(2) \in D_{1}(\delta), k=0,1,2, \ldots\right\} .
$$

Then

$$
D_{2}(\delta) \supset D_{1}(\delta) \supset \phi_{\delta}\left(\mathbf{Z}_{+}^{n}\right)
$$

and $D_{2}(\delta)$ has the following properties:

(1) $h(j) \in D_{2}(\delta), j=1,2, \ldots, n$ if $h \in D_{2}(\delta)$, in particular, $h(1,2) \in D_{2}(\delta)$;

(2) $h(1,2)+k e_{1}+s e_{2} \in D_{2}(\delta), k, s=0,1,2, \ldots$, if $h(1,2) \in D_{2}(\delta)$;

(3) If $h \in D_{2}(\delta)$, then there exists $k_{j}, 1 \leq k_{j} \leq\left\{\alpha_{j}\right\}$, such that $h+k_{j} e_{j} \in D_{2}(\delta)$, $j=3,4, \ldots, n$.

Using the same method as we used to prove (34), we can prove

$$
\begin{aligned}
K_{\alpha}(u) & >c_{19} \sum_{\delta \in Q} \sum_{h \in \mathbf{Z}_{+}^{n}} \bar{N}_{\delta}(2, h) B_{h, n}^{n+1, \alpha-1} u^{h} \prod_{j=3}^{n}\left(\frac{\sigma(h)+\sigma(\alpha)+1}{h_{j}+1} u_{j}\right)^{\varepsilon_{j}\left(\delta_{j}, h_{j}\right)} \\
& \times\left(\frac{\sigma(h)+\sigma(\alpha)+1}{h_{1}+1} u_{1}\right)^{\varepsilon_{1}^{\prime}\left(\delta_{1}, h_{1}\right)}\left(\frac{\sigma(h)+\sigma(\alpha)+1}{h_{2}+1} u_{2}\right)^{\varepsilon_{2}^{\prime}\left(\delta_{2}, h_{2}\right)}
\end{aligned}
$$

where $c_{19}$ is a positive constant, which only depends on $\alpha$ and $n$, and $\bar{N}_{\delta}(2, h)$ is the characteristic function of $D_{2}(\delta)$, that is, $\bar{N}_{\delta}(2, h)=1$ if $h \in D_{2} ; \bar{N}_{\delta}(2, h)=0$ if $h$ does not belong to $D_{2}$; and $\varepsilon_{2}^{\prime}\left(\delta_{2}, h_{2}\right)$ has the following property:

$$
\varepsilon_{2}^{\prime}\left(1, h_{2}\right) \geq 0, \quad \varepsilon_{2}^{\prime}\left(0, h_{2}\right) \leq 0 .
$$

We can repeat this process again and again, finally, we have

$$
\begin{aligned}
K_{\alpha}(u)> & c_{20} \sum_{\delta \in Q} \sum_{h \in \mathbf{Z}_{+}^{n}} \bar{N}_{\delta}(n, h) B_{h, n}^{n+1, \alpha-1} u^{h} \\
& \times \prod_{j=1}^{n}\left(\frac{\sigma(h)+\sigma(\alpha)+1}{h_{j}+1} u_{j}\right)^{\varepsilon_{j}^{\prime}\left(\delta_{j}, h_{j}\right)}
\end{aligned}
$$

where $c_{20}$ is a positive constant, which only depends on $\alpha$ and $n$, and $\bar{N}_{\delta}(n, h)$ is the characteristic function of $D_{n}(\delta)$, by the definition of $D_{j}(\delta), j=1,2, \ldots, n$, we have $D_{n}(\delta)=\mathbf{Z}_{+}^{n}$, thus $\overline{\mathbf{N}}_{\delta}(n, h) \equiv 1$, when $h \in \mathbf{Z}_{+}^{n}$; and $\varepsilon_{j}^{\prime}\left(\delta_{j}, h_{j}\right)$ has the properties: 


$$
\varepsilon_{j}^{\prime}\left(1, h_{j}\right) \geq 0, \quad \varepsilon_{j}^{\prime}\left(0, h_{j}\right) \leq 0, \quad j=1,2, \ldots, n .
$$

Thus

$$
K_{\alpha}(u)>c_{20} \sum_{h \in \mathbf{Z}_{+}^{n}} B_{h, n}^{n+1, \alpha-1} u^{h} \sum_{s \in Q} \prod_{j=1}^{n}\left(\frac{\sigma(h)+\sigma(\alpha)+1}{h_{j}+1} u_{j}\right)^{\varepsilon_{j}^{\prime}\left(\delta_{j}, h_{j}\right)} .
$$

For a fixed $h \in \mathbf{Z}_{+}^{n}$, we may take $\delta=\left(\delta_{1}, \ldots, \delta_{n}\right) \in Q$, such that

$$
\begin{array}{ll}
\delta_{j}=0 & \text { if } \frac{\sigma(h)+\sigma(\alpha)+1}{h_{j}+1} u_{j} \leq 1 ; \\
\delta_{j}=1 & \text { if } \frac{\sigma(h)+\sigma(\alpha)+1}{h_{j}+1} u_{j} \geq 1 ;
\end{array}
$$

then

$$
\prod_{j=1}^{n}\left(\frac{\sigma(h)+\sigma(\alpha)+1}{h_{j}+1} u_{j}\right)^{\varepsilon_{j}^{\prime}\left(\delta_{j}, h_{j}\right)} \geq 1
$$

and hence

$$
\sum_{\delta \in Q} \prod_{j=1}^{n}\left(\frac{\sigma(h)+\sigma(\alpha)+1}{h_{j}+1} u_{j}\right)^{\varepsilon_{j}^{\prime}\left(\delta_{j}, h_{j}\right)} \geq 1 .
$$

Finally we obtain

$$
K_{\alpha}(u)>c_{20} \sum_{h \in \mathbf{Z}_{+}^{n}} B_{h, n}^{n+1, \alpha-1} u^{h}=c_{20} F_{n}^{n+1, \alpha-1}(u)=c_{20} F(z, \bar{z}) .
$$

We have completed the proof of the Theorem.

\section{ACKNOWLEDGEMENTS}

This paper was written while the first author was at the Department of Mathematics of the University of California, San Diego, as a visiting professor. The first author is grateful to the department for their support, and also would like to express gratitude to Professor John D'Angelo for helpful and important communications.

\section{REFERENCES}

1. B. Bergman, Über die Kernfunktion eines Bereiches und ihr Verhalten am Rande, J. Reine Angew. Math. 169 (1933), 1-42.

2. L. Boutet de Monvel and J. Sjöstrand, Sur la singularité des noyaux de Bergman et de Szegö, Astérisque 34-45, Soc. Math. de Paris, France, 1976, pp. 123-164. MR 58:28684

3. D. Catlin, Estimates of invariant metrics on pseudoconvex domains of dimension two, Math. Z. 200 (1989), 429-466. MR 90e:32029

4. J. P. D'Angelo, A note on the Bergman kernel, Duke Math. J. 45 (1978), 259-266. MR $\mathbf{5 7 : 1 2 9 0 6}$

5. 23-34. MR 95a:32039

6. C. Fefferman, The Bergman kernel and biholomorphic mappings of pseudoconvex domains, Invent. Math. 26 (1974), 1-65. MR 50:2562

7. J. McNeal, Boundary behavior of the Bergman kernel function in $\mathbf{C}^{2}$, Duke Math. J. 58 (1989), 499-512. MR 91c:32017 
8. L L L L Lal geometry of decoupled pseudoconvex domain, Aspekte der Math. E17 (1990), 223-230. MR 92g:32033

9. A. Nagel, J. P. Rosay, E. M. Stein, and S. Wainger, Estimates for the Bergman and Szegö kernels in $\mathbf{C}^{2}$, Ann. of Math. 129 (1989), 113-149. MR 90g:32028

Department of Mathematics, University of Science and Technology of China, Hefei, Anhui, 230026, People's Republic of China

Department of Mathematics, University of California, San Diego, La Jolla, CaliFORNIA 92093

Department of Mathematics, Anhui University, Hefei, Anhui, 230039, People's RePUBLIC OF CHINA 